\title{
Baade-Wesselink distances to Galactic and Magellanic Cloud Cepheids and the effect of metallicity ${ }^{\star}$
}

\author{
M. A. T. Groenewegen
}

Koninklijke Sterrenwacht van België, Ringlaan 3, 1180 Brussels, Belgium

e-mail: marting@oma.be

Received 26 September 2012 / Accepted 22 October 2012

ABSTRACT

Context. The metallicity dependence of the Cepheid period-luminosity (PL) relation is of importance in establishing the extragalactic distance scale.

Aims. The aim of this paper is to investigate the metallicity dependence of the PL relation in $V$ and $K$, based on a sample of 128 Galactic, 36 Large Magellanic Cloud (LMC), and 6 Small Magellanic Cloud (SMC) Cepheids with individual BaadeWesselink (BW) distances (some of the stars also have an Hubble Space Telescope (HST) based and HIPPARCos parallax or are in clusters) and individually determined metallicities from high-resolution spectroscopy.

Methods. Literature values of the $V$-band, $K$-band, and radial velocity data were collected for the sample of Cepheids. Based on a $(V-K)$ surface-brightness relation and a projection factor, distances were derived from a BW analysis.

Results. The $p$-relation finally adopted is $1.50-0.24 \log P$. The slope of this relation is based on the condition that the distance to the LMC does not depend on period or $(V-K)$ colour and that the slope of the PL relation based on the BW distances agrees with that based on apparent magnitude. The zero point of the relation is tight to the Cepheids with HST and revised HIPPARcos parallaxes as well as to Cepheids in clusters. The slope of the Galactic and LMC $K$-band relation formally agrees within the errors, and combining all Cepheids (including the SMC) results in a negligible metallicity dependence and a relation of $M_{K}=(-2.50 \pm 0.08)+(-3.06 \pm 0.06) \log P$. A similar conclusion is found for the reddening-free Wesenheit relation $(W(V K)=K-0.13(V-K))$, with $M_{\mathrm{WvK}}=(-2.68 \pm 0.08)+(-3.12 \pm 0.06) \log P$. In the $V$-band the situation is more complex. The slope of the LMC and the Galactic PL relation differ at the $3 \sigma$ level. Combining the sample nevertheless results in a metallicity term significant at the $2 \sigma$ level: $M_{\mathrm{V}}=(-1.55 \pm 0.09)+(-2.33 \pm 0.07) \log P+(+0.23 \pm 0.11)[\mathrm{Fe} / \mathrm{H}]$. Taking only the Galactic Cepheids, the metallicity term is no longer significant, namely $(+0.17 \pm 0.25)$. Compared to the recent works by Storm et al. (2011a, A\&A, 534, A94; 2011b, A\&A, 534, A95), there is both agreement and disagreement. A similar dependence of the $p$-factor on period is found, but the zero point found here implies a shorter distance scale. The distance modulus (DM) to the LMC and SMC found here are $18.29 \pm 0.02$ and $18.73 \pm 0.06$ (statistical error on the mean), respectively. Systematic differences in reddening could have an effect of order +0.05 in DM. The details of the comparison of BW-based distances and Cepheids with HST and revised HIPPARCos parallaxes also play a role. The method used by Storm et al. would lead to larger DM of 18.37 and 18.81 for the LMC and SMC, respectively. The LMC DM is shorter than the currently accepted value, which is in the range 18.42 to 18.55 , and it is speculated that the $p$-factor may depend on metallicity. This is not predicted by theoretical investigations, but these same investigations do not predict a steep dependence on period either, indicating that additional theoretical work is warranted.

Key words. stars: distances - stars: variables: Cepheids

\section{Introduction}

Cepheids are considered an important standard candle because they are bright and thus the link between the distance scale in the nearby universe and that further out via those galaxies that contain both Cepheids and SNIa (e.g. Riess et al. 2009)

Distances to local Cepheids may be obtained in several ways, e.g. through main-sequence fitting for Cepheids in clusters (e.g. Feast 1999; Turner 2010) or via determination of the parallax. Benedict et al. (2007) published absolute trigonometric parallaxes for ten Galactic Cepheids using the Fine Guidance Sensor on board the Hubble Space Telescope (HST), and revised HIPPARCos parallaxes have also become available (van Leeuwen et al. 2007).

In addition, distances to Cepheids can be obtained from the Baade-Wesselink (BW) method. This method relies on the availability of surface-brightness (SB) relations to link variations in colour to variations in angular diameters and an understanding

* Tables 1-6, 10, and Appendix A are available in electronic form at http://www. aanda.org of the projection $(p-)$ factor, which links radial velocity to pulsational velocity variations.

A lot has been published on the subject over the past decade by the group of Storm/Gieren/Fouqué and coworkers (Storm et al. 2004a,b; Gieren et al. 2005; Fouqué et al. 2007), which culminated in the recent works by Storm et al. (2011a,b). In those two papers, the authors analysed 70 Galactic and 41 Magellanic Cloud (MC) Cepheids. They found that, (a) the $p$-factor depends quite steeply on period, confirming Gieren et al. (2005), based on the requirement that the distance to the (barycenter of the) Large Magellanic Cloud (LMC) should not depend on period; (b) the $K$-band period-luminosity (PL) relation is universal, $M_{K}=-3.30( \pm 0.06)(\log P-1)-5.65( \pm 0.02)$; (c) the $K$-band PL relation is insensitive to metallicity; and (d) a distance modulus to the barycenter of the LMC of $18.45 \pm 0.04$ for a $p$-factor relation $1.550-0.186 \log P$, where the zero point was calibrated on the Cepheids with HST parallaxes (Benedict et al. 2007).

Independently, Groenewegen (2007, hereafter G07) investigated the SB relation (finding excellent agreement with the relation by Kervella et al. 2004a) and the $p$-factor, based on six 
Cepheids with interferometrically measured angular-diameter variations and known distances. Groenewegen (2008, hereafter G08) presented BW distances to 68 Galactic Cepheids with individually determined metallicities from high-resolution spectroscopy.

The main aim of that particular work was to address the metallicity dependence of the PL relation, which remains a matter of debate. Observations seem to consistently indicate that metal-rich Cepheids are brighter, and various estimates have been given in the literature, $-0.88 \pm 0.16 \mathrm{mag} / \mathrm{dex}(B R I$ bands, Gould 1994), $-0.44_{-0.2}^{+0.1} \mathrm{mag} / \mathrm{dex}$ (VR bands, Sasselov et al. 1997), $-0.24 \pm 0.16 \mathrm{mag} / \mathrm{dex}$ (VI bands, Kochanek 1997), $-0.14 \pm 0.14 \mathrm{mag} / \mathrm{dex}$ ( $V I$ bands, Kennicutt et al. 1998), $-0.21 \pm$ 0.19 in $V,-0.29 \pm 0.19$ in $W,-0.23 \pm 0.19$ in $I,-0.21 \pm$ $0.19 \mathrm{mag} / \mathrm{dex}$ in $K$ (Storm et al. 2004a,b), $-0.29 \pm 0.10 \mathrm{mag} / \mathrm{dex}$ ( $B V I$ bands, Macri et al. 2006). G08 found values of $+0.27 \pm$ $0.30 \mathrm{mag} / \mathrm{dex}(V$-band) and $-0.11 \pm 0.24 \mathrm{mag} / \mathrm{dex}(K$-band).

Since then, values have been reported of $-0.29 \pm$ $0.11 \mathrm{mag} / \mathrm{dex}$ ( $W_{\mathrm{VI}}$, Scowcroft et al. 2009), and a very steep value of $\sim-0.8 \pm \sim 0.2\left(W_{\mathrm{VI}}\right.$, Shappee \& Stanek 2011). Finally, Storm et al. (2011) quote slopes of $-0.23 \pm 0.10 \mathrm{mag} / \mathrm{dex}\left(W_{\mathrm{VI}}\right)$, $+0.09 \pm 0.10 \mathrm{mag} / \mathrm{dex}(V$-band $)$, and $-0.10 \pm 0.10 \mathrm{mag} / \mathrm{dex}$ ( $K$-band).

With the exception of Groenewegen (2008), no individual abundance determinations of individual Cepheids are used in these studies. Instead, abundances of nearby HII regions or even a mean abundance of the entire galaxy are used. Even in the recent work by Storm et al. all Galactic, Small Magellanic Cloud (SMC) and LMC Cepheids were assigned a metallicity typical of that galaxy.

The present paper is an update of Groenewegen $(2007,2008)$ and takes into account the latest available data, in terms of interferometrically determined angular diameters, photometric and radial velocity data, and individually determined metallicities. In this paper, we revisit BW distances to Cepheids with metallicity determinations, increasing the sample to over 120 Galactic stars, adding 6 SMC and 42 LMC objects, and using updated $p$-factor and SB relations. Section 2 describes the selection of the photometric and radial velocity data. Section 3 outlines how the data are modelled. In Sect. 4, the surface-brightness relation is discussed. Section 5 describes how the binary Cepheids are treated, and new and updated orbital elements are presented. Section 6 describes the results regarding the period-luminosity (-metallicity) (PL(Z)) relation. Section 7 presents conclusions.

\section{The sample}

Table 1 lists the 128 Galactic classical Cepheids used in this study. This is a significant increase with respect to the 68 stars considered in G08. This sample represents essentially all Galactic Cepheids with accurate individually determined metallicities (mostly from Luck \& Lambert 2011 and Luck et al. 2011) that have sufficient optical, $K$-band, and radial velocity data available for a BW analysis ${ }^{1}$. The single largest new dataset that made this increase in sample size possible is the recent publication of near-infrared light curves of 131 northern Cepheids by Monson \& Pierce (2011).

Contrary to G08, 42 MC Cepheids are also considered here, and Table 2 lists that sample. The radial velocity data presented by Storm et al. (2011b) contribute significantly to the

\footnotetext{
1 Some Cepheids have considered, but the datasets were deemed insufficient for an accurate BW analysis: BK Aur and GH Cyg (too poor radial velocity data), EV Sct and X Sct (too poor NIR data).
}

fact that a BW analysis is now feasible for a sizeable sample of MC Cepheids.

Tables 1 and 2 list the sources of the $V$-band, $K$-band, and radial velocity data, as well as data which were not considered in the BW analysis ${ }^{2}$. Sometimes a certain range in Julian date is excluded, mostly to exclude some of the older datasets, which are less accurate. This is also done because there is a clear change in pulsation period, and these cases are marked by $\dot{P}$.

Since 2008, additional radial velocity data has been obtained using the $1.2 \mathrm{~m}$ Euler telescope located at the La Silla observatory (see G08, for a description of the data taking and data reduction). The new radial velocity (RV) data are presented in Table 3.

Tables 4 and 5 list the published iron abundances for the Galactic Cepheids and for 15 MC Cepheids (only one of those, HV 837, is located in the SMC). Most Galactic $[\mathrm{Fe} / \mathrm{H}]$ values come from Luck \& Lambert (2011) and Luck et al. (2011), while the majority of MC Cepheid determinations are from Romaniello et al. (2008). To put all metallicities on the scale of Luck \& Lambert (2011), differences were determined between the other references and them. Following the reference numbers in Table 4, the differences are $2-1=-0.07 \pm 0.09$ ( $N=184$ objects in common), $3-1=-0.11 \pm 0.11(N=25)$, $4-1=-0.18 \pm 0.08(N=11)$, and $5-1=-0.09 \pm 0.07$ $(N=47)$. The $[\mathrm{Fe} / \mathrm{H}]$ values used in this paper are the published values corrected for these offsets.

For the five SMC Cepheids without metallicity determination the value in Storm et al. (2011b) is adopted: $[\mathrm{Fe} / \mathrm{H}]=-0.68$. For the four Cepheids in the LMC cluster NGC 1866 without a metallicity determination a value of -0.39 is adopted. This is the median of the values of the three Cepheids in the cluster that do have a metallicity determination (HV 12197, HV 12199, and We2; Molinaro et al. 2012) and is in agreement with the average metallicity of -0.43 determined by Mucciarelli et al. (2011). For the other LMC Cepheids without metallicity determination, the value used in Storm et al., -0.34 is used. This is very close to the median value of -0.36 of the 12 LMC Cepheids not in NGC 1866 listed in Table 5.

Reddening values with errors were preferentially taken from Fouqué et al. (2007, for 105 objects) and otherwise from van Leeuwen et al. (2007, for 16 objects, the value listed as ET in their Table A1), Andrievsky et al. (2002, four objects), Luck et al. (2011, one object), and Tammann et al. (2004, two objects). For the MC Cepheids, the values in Storm et al. (2011b) were adopted with an error in $E(B-V)$ of 0.005 . The $E(B-V)$ values and errors are listed in Col. 2 of Table 10.

The following Galactic Cepheids are considered to be first overtone pulsators (e.g. Klagyivik \& Szabados 2009): FF Aql, SZ Tau, SU Cas, QZ Nor, GH Lup, DT Cyg. When relations are plotted against period, their periods are "fundamentalised" following Alcock et al. (1995). None of the LMC and SMC Cepheids in the sample are believed to be overtone pulsators.

\section{The model}

The model is outlined in G07 but will be briefly repeated here. The $V$-, $K$ - and RV data with error bars are fitted with a function of the form

$F(t)=F_{0}+\sum_{i=1}^{i=N}\left(A_{i} \sin \left(2 \pi t \mathrm{e}^{\mathrm{i} f}\right)+B_{\mathrm{i}} \cos \left(2 \pi t \mathrm{e}^{\mathrm{i} f}\right)\right)$,

2 When deriving the binary orbits, all radial velocity (RV) data were used (see Sect. 5). 
where $P=\mathrm{e}^{-f}$ is the period (in days). The period is determined from the fit to the available optical photometry as this dataset is usually the most extensive. The period is then fixed when fitting Eq. (1) to the $K$-band and RV data.

The determination of the parameters is done using the MRQMIN routine (following the Levenberg-Marquardt method) from Press et al. (1992), which minimises

$\chi^{2}=\sum_{i=1}^{i=n}\left(F_{i}-F\left(t_{i}\right)\right)^{2} /\left(\sigma_{\mathrm{F}_{\mathrm{i}}}\right)^{2}$,

with $F_{i}$ the measurement at time $t_{i}$, which has an error bar $\sigma_{F_{i}}$. Also the reduced $\chi^{2}$ is defined as

$\chi_{\mathrm{r}}^{2}=\frac{\chi^{2}}{(n-p)}$,

and the quantity BIC as

$\mathrm{BIC}=\chi^{2}+(p+1) \ln (n)$,

where $p=2 N+2$ is the number of free parameters $(p=2 N+1$ when fitting the RV and $K$ light curve). As the number $N$ of harmonics to be fitted to the data is a priori not known, one could obtain ever better fits (lower $\chi^{2}$ ) by increasing $N$. The Bayesian information criterion (Schwarz 1978) is a formalism that penalises this, and $N$ (for the $V, K$ and RV curve independently) is chosen such that BIC reaches a minimum. The number of harmonics used varies between 3 and 10 in the optical, 1 and 5 in the NIR, and 2 and 8 for the RV curves.

Given the analytical form of Eq. (1), the RV curve can be exactly integrated to obtain the variation in radius as a function of time (phase):

$\Delta R(t, \delta \theta)=-p \int_{t_{0}}^{t+P \delta \theta}\left(v_{\mathrm{R}}-\gamma\right) \mathrm{d} t$,

where $\gamma$ is the systemic velocity, $v_{\mathrm{R}}$ the radial velocity, $p$ the projection factor and $\delta \theta$ allows for a phase shift between the RV curve and the angular-diameter variations determined via the $\mathrm{SB}$ relation.

Then, the equation

$\theta(t)=9.3038 \operatorname{mas}\left(\frac{R_{0}+\Delta R(t, \delta \theta)}{d}\right)$

is fitted with $\theta$ the angular diameter in mas, $R_{0}$ the stellar radius in solar radii and $d$ the distance in pc.

Compared to G08, the fitting procedure was changed. In G08, the fitting was done implementing the linear bisector (using the code SIXLIN from Isobe et al. 1990) as used and preferred by e.g. Storm et al. (2004a,b), Barnes et al. (2005a,b), Gieren et al. (2005), and Storm et al. (2011a,b). The bisector is still the preferred method, but errors in both $\Delta R$ and $\theta$ are now taken into account using the bivariate correlated errors and intrinsic scatter (BCES) method (Akritas \& Bershady 1996) ${ }^{3}$. The error in $\theta(t)$ includes the error in $V$ and $K$ magnitude and the error in $E(B-V)$, while the error in $\Delta R$ includes the error in the Fourier coefficients, see Eq. (1). An alternative method is also considered based on a non-linear least-squares fit with four parameters $(p$, $d, R_{0}$, and $\delta \theta$ ). When the distance is known (the Cepheids with HST parallaxes, see Sect. 4), one solves for $p\left(R_{0}\right.$ and $\left.\delta \theta\right)$. In most cases (Sect. 6), one solves for $d\left(R_{0}\right.$ and $\left.\delta \theta\right)$ for a given $p$. In

\footnotetext{
3 http://www . astro.wisc. edu/ mab/archive/stats/stats. html
}

G08, this method was already implemented in order to derive $\delta \theta$. Then the bisector method was used with this $\delta \theta$ in order to derive the distance. However, the value of $\delta \theta$ that best fits the data from the non-linear fit does not necessarily best fit the data using the bisector. In the present paper, the non-linear fit is used to derive $\delta \theta$ and its error, and the BCES method is used for 21 values of $\delta \theta$ within $\pm 4 \sigma$ of its best-fit value to find the best-fitting distance. In some cases, a phase range around $0.85-0.95$ is excluded from the fit (likely related to shocks in the stellar atmosphere close to minimum radius).

\section{The $p$-factor and surface-brightness relation}

One way of deriving the $p$-factor (and its dependence on period) is to use interferometrically determined angular diameters for a sample of stars with known distances, e.g. Mérand et al. (2005) and G07. The conclusion in G07 was that, statistically, there was no need to include a period dependence and that a constant value of $p=1.27 \pm 0.05$ fitted the available data at that time, based on six stars.

The theoretical investigation by Nardetto et al. (2007) suggested that there is a difference between the $p$-factor to be used with wide-band interferometry (like in G07) and with RV data (when applying the SB technique as in G08 and the present study). For $\delta$ Cep, this difference is of the order of 0.06 (Nardetto et al. 2007), in the sense that in SB studies the $p$-factor is slightly larger and $p=1.33$ was used in G08.

New interferometric angular-diameter determinations have become available since 2008 , and the $p$-factor dependence on period is investigated first. Table 6 lists the stars with an independent distance estimate and/or interferometrically determined (limb-darkened) angular diameters. Columns 2 and 4 give the period and $E(B-V)$ value.

The distances come primarily from van Leeuwen et al. (2007), who took the weighted average of the HST determined parallax (Benedict et al. 2007) and the revised HIPPARCos parallax given in the same paper (the exception was Y Sgr for which the HST value was adopted). The Lutz-Kelker (LK) correction (Lutz \& Kelker 1973) in Col. 4 is taken from van Leeuwen et al. (2007) and included in determining the most probable distance. For $\delta$ Cep and $\zeta$ Gem, the recent distance determinations to the host clusters (Majaess et al. 2012a,b) were also considered in the distances finally adopted, which are listed in Col. 6. Column 7 lists the references to the interferometric observations.

Columns 8 and 9 list the derived $p$-factor and mean radius. The first error bar is the error in the fit, while the second is the error due to the error in the distance.

The analysis of these eight stars allows one to derive a period-radius (PR) relation, which is shown in Fig. 1. A linear weighted least-squares fit results in

$\log R=(0.696 \pm 0.033) \log P+(1.115 \pm 0.030), \sigma=0.022$,

where the two error bars for $R$ listed in Table 6 have been added quadratically. Figure 1 shows the recent PR relation from Molinaro et al. (2011), $\log R=(0.75 \pm 0.03) \log P+(1.10 \pm 0.03)$, for comparison.

Using the PR relation of Eq. (7), the radii of $\eta$ Aql and Y Oph were estimated with their error bar. For this radius, the distance and $p$-factor were determined with their error bars (the internal error is listed first, and then the error due to the uncertainty in $R$ ).

Figure 2 plots the relation between the derived $p$-factor and $\log P$ for the seven stars with an error (the quoted error bars on $p$ were added quadrature) less than 0.4. A weighted least-squares 


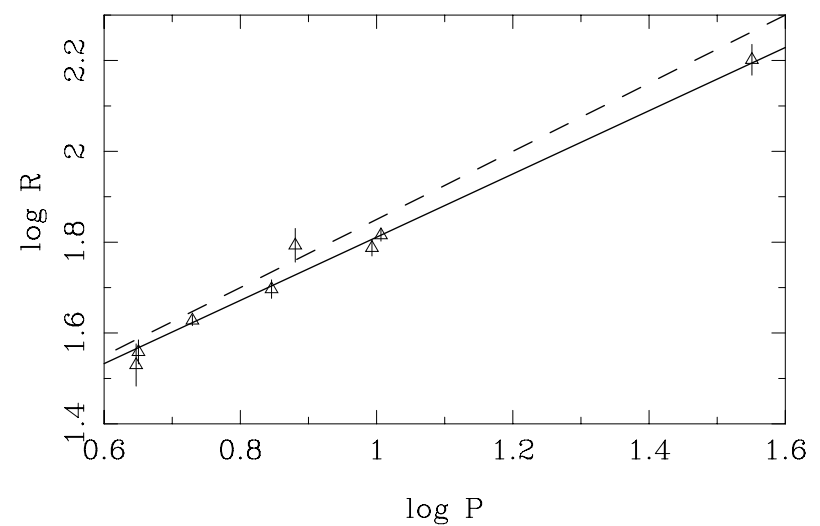

Fig. 1. The PR relation derived in the present paper (the solid line, which is the fit to the data points with error bars) compared to the PR relation of Molinaro et al. (2011), represented by the dashed line.

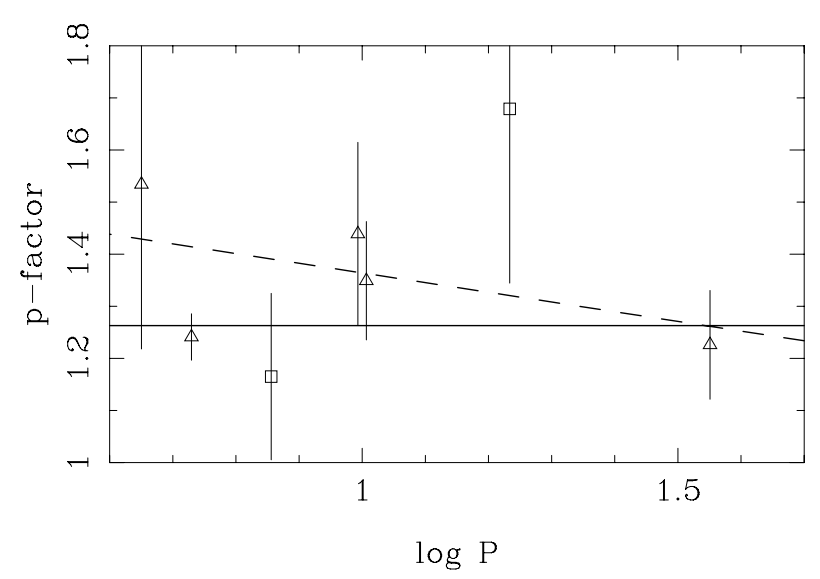

Fig. 2. The $p$-factor plotted versus log period for the seven stars that have an error in $p$ smaller than 0.4. The open squares indicate the two stars for which the radius was fixed from the PR relation. Also plotted is a line indicating the best constant value of $p=1.264$, while the dashed lines represent the $p$-factor relation proposed by Storm et al. (2011a), $p=1.550-0.186 \log P$.

fit is made to find that there is no evidence from this data alone of a dependence on period: $p=(1.24 \pm 0.12)+(+0.03 \pm 0.13) \log P$, nor on period and metallicity: $p=(1.75 \pm 0.40)+(+0.06 \pm$ $0.13) \log P+(-4.5 \pm 3.4)[\mathrm{Fe} / \mathrm{H}]$. The best-fitting constant value is $p=1.264 \pm 0.036$, similar to what was found in G07.

Independently of the derivation of the PR relation or the $p$-factor, the available interferometric, optical, and infrared data can be used to calibrate the SB relation for Cepheids, very much in line with Kervella et al. (2004a) and G07. An SB relation can be defined as follows (see van Belle 1999):

$\theta_{0}=\theta \times 10^{\left(m_{1} / 5\right)}$,

where $\theta$ is the LD angular diameter (in mas) and $m_{1}$ a dereddened magnitude (for example, $V$ ). The logarithm of this quantity (the zero magnitude angular diameter) is plotted against a de-reddened colour (for example, $(V-K)_{0}$ ),

$\log \theta_{0}=a\left(m_{2}-m_{3}\right)+b$.

The aim is to determine the coefficients $a$ and $b$.

Table 7 list the various results. Figure 3 shows the results, including all objects and where the error in the angular-diameter determination is less than $0.2^{\prime \prime}$ to exclude some extreme outliers. Different stars are marked by different symbols as given in the

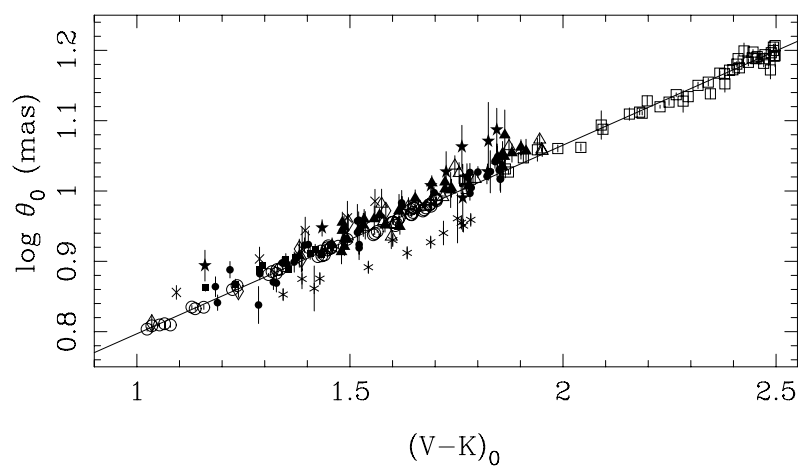

Fig. 3. $\log \theta_{0}$ versus de-reddened $(V-K)$ colour for Cepheids. Different symbols indicate the different stars. $(\delta \mathrm{Cep}=0, l \mathrm{Car}=\square, \zeta \mathrm{Gem}=\Delta$, $\eta \mathrm{Aql}=\bullet, \beta$ Dor $=\boldsymbol{\Delta}, \mathrm{FF} \mathrm{Aql}=\mathbf{\square}, \mathrm{T} \mathrm{Vul}=\diamond, \mathrm{W} \mathrm{Sgr}=\star, \mathrm{X} \mathrm{Sgr}=\mathrm{x}$,

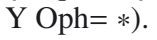
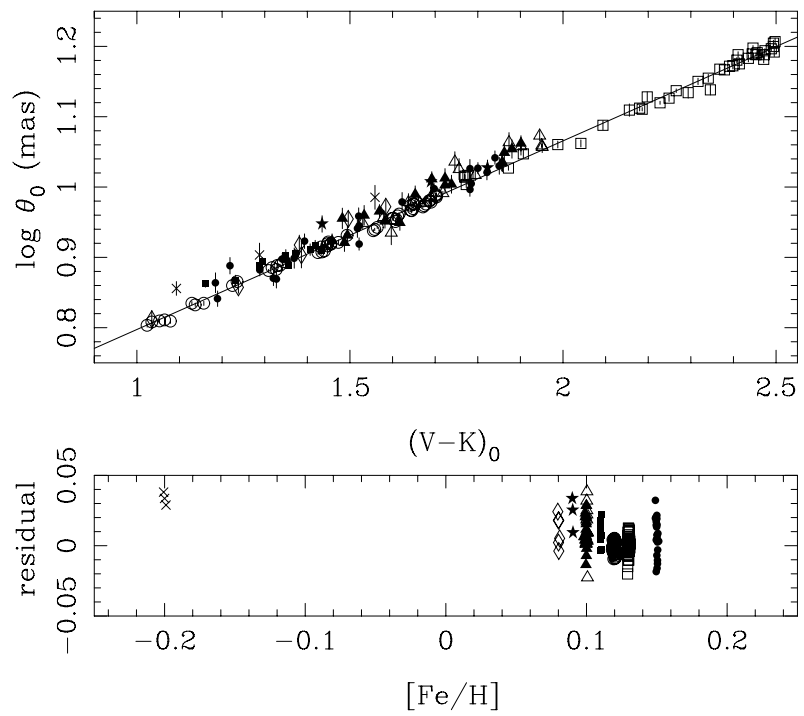

Fig. 4. $\log \theta_{0}$ versus $(V-K)_{0}$ colour for Cepheids, excluding Y Oph and with errors on the angular diameters $<0.065^{\prime \prime}$. The bottom panel shows the residuals plotted against $[\mathrm{Fe} / \mathrm{H}]$. Symbols as Fig. 3.

caption. The relation is extremely well defined but as noted in G07 Y Oph (the * -symbol) clearly lies below the relation, and there are still some data points with large error bars (e.g. W Sgr, indicated by the $\star$ symbol). The determination of the SB relation is affected by reddening, and Y Oph has by far the largest reddening of the stars under consideration here. A reddening of $E(B-V) \sim 1$ would bring it onto the relation.

Figure 4 shows the results ignoring $\mathrm{Y}$ Oph and selecting only angular diameters determined with an error $<0.065^{\prime \prime}$. More complicated relationships than Eq. (9) were also investigated, in particular adding a quadratic term in $(V-K)_{0}^{2}$, a linear term on period, and a linear term in $[\mathrm{Fe} / \mathrm{H}]$. The quadratic term in colour and a linear term on period do not result in coefficients that are significant. However, a linear term on metallicity (i.e. $\log \theta_{0}=a(V-K)_{0}+b+c[\mathrm{Fe} / \mathrm{H}]$ appears significant, as illustrated in the bottom panel of Fig. 4.

This result does not depend on $\mathrm{X} \mathrm{Sgr}$ at $[\mathrm{Fe} / \mathrm{H}]=-0.20$. Excluding it still results in a coefficient determined at the $3 \sigma$ level. Uncertainties in reddening could introduce a metallicity dependence. When FF Aql is ignored as well, thereby restricting the sample to the stars with $E(B-V)$ less than 0.15 , and only angular diameters determined with an error $<0.05^{\prime \prime}$ are 

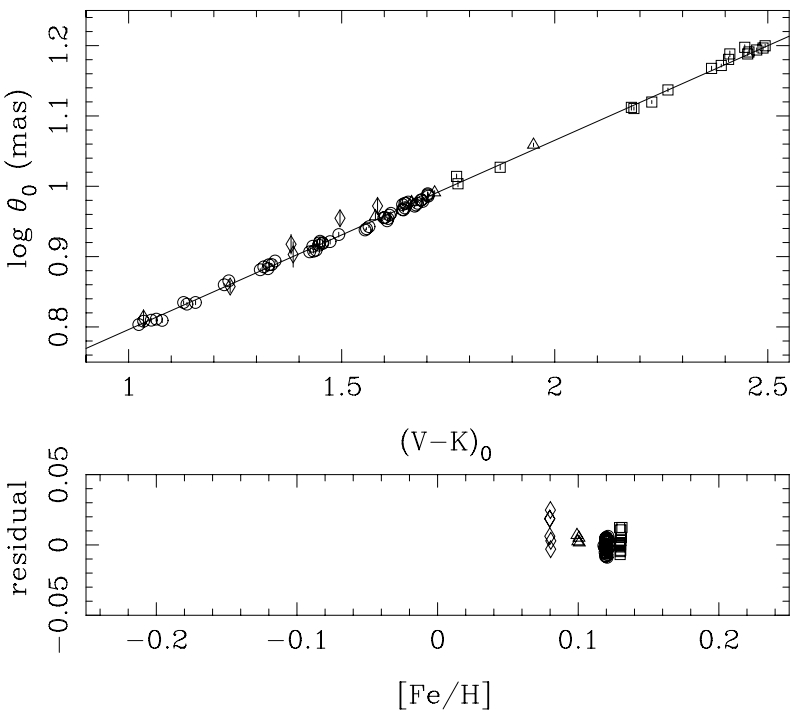

Fig. 5. $\log \theta_{0}$ versus $(V-K)_{0}$ colour for Cepheids, excluding Y Oph, $\mathrm{X}$ Sgr and FF Aql and with errors on the angular diameters $<0.05^{\prime \prime}$. The bottom panel shows the residuals plotted against $[\mathrm{Fe} / \mathrm{H}]$. Symbols as Fig. 3.

selected, the coefficient is determined at the the $2 \sigma$ level (see Fig. 5).

Excluding X Sgr, the spread in $[\mathrm{Fe} / \mathrm{H}]$ over the sample is small and comparable to the measurement error. To test a possible metallicity dependence of the SB relation, a Monte Carlo simulation was performed, assuming a Gaussian error of 0.05 dex in $[\mathrm{Fe} / \mathrm{H}]$ and Gaussian errors in $E(B-V), V$, and $K$. The resulting SB relations are reported in Cols. 8-12 of Table 7. The dependence on metallicity is weaker now, at the $1 \sigma$ level or less.

The SB relation adopted in this paper is based on the second solution from Table 7, $\log \theta_{0}=0.2674(V-K)_{0}+0.5327$. Table 7 also includes other SB relations, including the one by Kervella et al. (2004a), which has also been used by Storm et al. $(2011 a, b)^{4}$. The agreement is excellent.

Figure 6 compares the angular diameters determined from interferometry with those calculated from photometry and the $\mathrm{SB}$ relation for $\delta$ Cep and $l$ Car, the two Cepheids with the best and most extensive set of interferometrically determined angular diameters. The agreement is very good and illustrates the power of SB relations.

\section{Binary Cepheids}

A number of stars in the sample are known or suspected spectroscopic binaries. In order to apply the SB technique outlined in the previous section, the RV data have to be corrected for the binary motion. The procedure is outlined in G08. For a number of stars ${ }^{5}$, the elements of G08 were used. For FF Aql, V350 Sgr, V496 Aql, and VZ Cyg, new RV data allowed for an improved orbit compared to G08. For X Sgr, the new RV data do not support the solution presented in G08. Although a period analysis continues to show a peak at 572 days, the binary solution is not satisfactory. For X Sgr no binary orbit is assumed here. The

\footnotetext{
4 Note that in their 2011a paper near their Eq. (2), it is stated that the SB relation used is from Fouque \& Gieren (1997) but this is not the case. The $2011 \mathrm{~b}$ paper has the correct reference.

5 S Mus, S Nor, S Sge, SU Cas, SU Cyg, T Mon, U Vul, W Sgr, XX Cen, Z Lac.
}
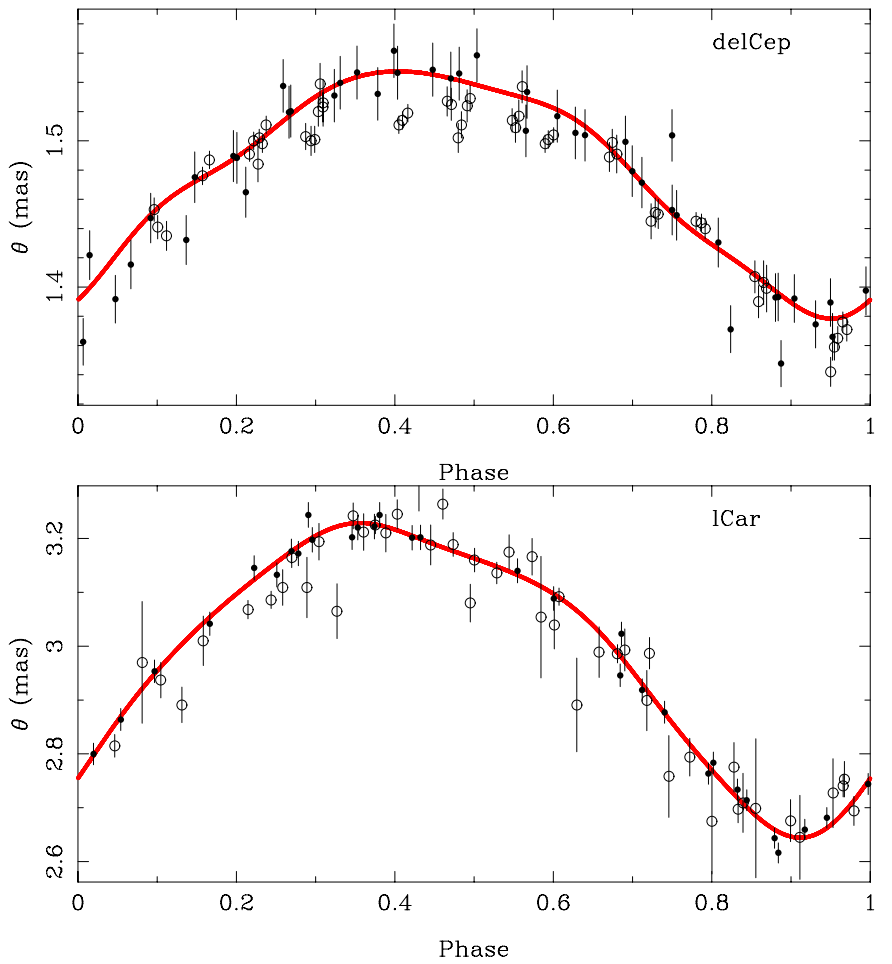

Fig. 6. Comparison between the angular diameters determined from the SB relation (filled circles), and from interferometry (open circles) from $\delta$ Cep and $l$ Car.

orbital elements (three updated and five new orbits) are listed in Table 8.

For V496 Aql the period changed considerable. A period analysis showed that the period preferred in G08 (1331 days) only has the third highest peak in the current Fourier spectrum and that 1065 days clearly shows the better fit. For V350 Sgr the orbit is updated and compares well to the recent orbit by Evans et al. (2011).

For the known spectroscopic binaries, the derived orbital parameters are close to literature values, cf. RX Cam (Imbert 1996), DL Cas (Gieren et al. 1994), MW Cyg (Imbert 1996; and Rastorgouev et al. 1997. The eccentricity derived here is in between the two values quoted), AW Per (Evans et al. 2000), and U Aql (Welch et al. 1987a,b). For Y Oph, the very non-eccentric orbit proposed by Abt \& Levy (1978) is not confirmed.

\section{Results}

\subsection{The period dependence of the p-factor}

In their most recent work, Storm et al. (2011a) proposed a $p$-factor of $(1.55 \pm 0.04)-(0.186 \pm 0.06) \log P$, confirming their earlier result of $p=(1.58 \pm 0.02)-(0.15 \pm 0.05) \log P$ by Gieren et al. (2005). The slope is derived from the requirement that the distance to the barycenter of the LMC should not depend on period.

Independently, for the Galactic Cepheids with HST parallaxes, one can determine the $p$-factor which makes the BW distance equal to the HST-based distance. Storm et al. (2011a) find $p=(1.65 \pm 0.07)-(0.28 \pm 0.08) \log P$. The $p$-factors derived in this way are reported in Col. 10 of Table 6 and a fit to the six stars with and error $<0.15$ gives $p=(1.33 \pm 0.16)-(0.07 \pm 0.16) \log P$. The best-fit constant value is $p=1.27 \pm 0.03$. In a similar fashion, Ngeow et al. (2012) considered not only stars with an 
Table 7. Coefficients of the SB relation.

\begin{tabular}{|c|c|c|c|c|c|c|c|c|c|c|c|}
\hline Condition & $N$ & $b$ & $a$ & $b$ & $a$ & $c$ & $b$ & $a$ & $b$ & $a$ & $c$ \\
\hline \multirow[t]{2}{*}{$\sigma_{\theta}<0.2$} & \multirow[t]{2}{*}{226} & 0.5322 & 0.2672 & 0.5435 & 0.2689 & -0.119 & 0.5335 & 0.2663 & 0.5382 & 0.2667 & -0.056 \\
\hline & & 0.0032 & 0.0019 & 0.0039 & 0.0019 & 0.022 & 0.0029 & 0.0017 & 0.0078 & 0.0039 & 0.048 \\
\hline$\sigma_{\theta}<0.065$ & \multirow[t]{2}{*}{170} & 0.5327 & 0.2674 & 0.5435 & 0.2688 & -0.111 & 0.5338 & 0.2667 & 0.5384 & 0.2664 & -0.048 \\
\hline Y Oph excluded & & 0.0033 & 0.0020 & 0.0043 & 0.0020 & 0.028 & 0.0028 & 0.0016 & 0.0073 & 0.0032 & 0.043 \\
\hline$\sigma_{\theta}<0.065$ & \multirow[t]{2}{*}{167} & 0.5317 & 0.2679 & 0.5479 & 0.2691 & -0.151 & 0.5329 & 0.2672 & 0.5370 & 0.2662 & -0.032 \\
\hline Y Oph \& X Sgr excluded & & 0.0033 & 0.0020 & 0.0070 & 0.0020 & 0.057 & 0.0029 & 0.0016 & 0.0099 & 0.0035 & 0.069 \\
\hline$\sigma_{\theta}<0.03$ & \multirow[t]{2}{*}{79} & 0.5251 & 0.2705 & 0.5477 & 0.2723 & -0.212 & 0.5263 & 0.2697 & 0.5319 & 0.2679 & -0.030 \\
\hline Y Oph, X Sgr, \& FF Aql excluded & & 0.0042 & 0.0026 & 0.0133 & 0.0028 & 0.119 & 0.0038 & 0.0020 & 0.0120 & 0.0030 & 0.096 \\
\hline \multirow[t]{2}{*}{ Fouqué \& Gieren (1997) } & & 0.547 & 0.262 & & & & & & & & \\
\hline & & 0.006 & 0.004 & & & & & & & & \\
\hline \multirow[t]{2}{*}{ Kervella et al. (2004a) } & & 0.5354 & 0.2672 & & & & & & & & \\
\hline & & 0.0012 & 0.0016 & & & & & & & & \\
\hline \multirow[t]{2}{*}{ G07 } & & 0.5235 & 0.2752 & & & & & & & & \\
\hline & & 0.0092 & 0.0045 & & & & & & & & \\
\hline
\end{tabular}

Notes. Column 1 gives the conditions applied or the reference to the literature; Col. 2 the number of data points, and Cols. 3-4 and 5-7 the coefficients of the SB relation. Columns 8-12 repeat these numbers based on the Monte Carlo simulation. The second line gives the errors in the coefficients. The adopted coefficients are indicated in boldface.

Table 8. Derived orbital parameters of binary Cepheids.

\begin{tabular}{lcccccc}
\hline \hline Name & $\gamma\left(\mathrm{km} \mathrm{s}^{-1}\right)$ & $K\left(\mathrm{~km} \mathrm{~s}^{-1}\right)$ & $e$ & $\omega\left(^{\circ}\right)$ & $T_{0}(\mathrm{JD}-2400000)$ & Binary period $(\mathrm{d})$ \\
\hline FF Aql & $-15.71 \pm 0.07$ & $4.98 \pm 0.06$ & $0.011_{-0.008}^{+0.033}$ & $362 \pm 103$ & $45609 \pm 415$ & $1432.7 \pm 0.9$ \\
V496 Aql & $4.51 \pm 0.14$ & $3.63 \pm 0.18$ & 0 & 0 & $45480 \pm 17$ & $1066.2 \pm 1.9$ \\
V350 Sgr & $11.43 \pm 0.05$ & $10.50 \pm 0.07$ & $0.351_{-0.007}^{+0.007}$ & $283 \pm 1$ & $52015 \pm 4$ & $1468.9 \pm 0.9$ \\
VZ Cyg & $-16.96 \pm 0.10$ & $3.02 \pm 0.16$ & 0 & 0 & $44811 \pm 36$ & $2183 \pm 10$ \\
RX Cam & $-37.54 \pm 0.06$ & $14.27 \pm 0.11$ & $0.459_{-0.006}^{+0.007}$ & $78.4 \pm 1.0$ & $45931.3 \pm 1.8$ & $1113.8 \pm 0.5$ \\
DL Cas & $-36.23 \pm 0.06$ & $16.43 \pm 0.11$ & $0.350_{-0.006}^{+0.006}$ & $27.3 \pm 1.0$ & $47161.6 \pm 1.5$ & $684.27 \pm 0.16$ \\
MW Cyg & $-13.37 \pm 0.12$ & $6.43 \pm 0.19$ & $0.140_{-0.025}^{+0.030}$ & $78 \pm 13$ & $48862 \pm 15$ & $439.61 \pm 0.18$ \\
AW Per & $6.60 \pm 0.35$ & $12.06 \pm 0.32$ & $0.499_{-0.030}^{+0.032}$ & $254 \pm 3$ & $38721 \pm 178$ & $14293.70 \pm 283$ \\
U Aql & $1.14 \pm 0.14$ & $7.75 \pm 0.22$ & $0.134_{-0.021}^{+0.025}$ & $163 \pm 9.5$ & $42634 \pm 49$ & $1853.6 \pm 3.0$ \\
\hline
\end{tabular}

Notes. Quantities without error bar have been fixed.

HST parallax (from van Leeuwen et al. 2007) but also Cepheids in clusters (from Turner 2010), determining that $p$-factor that makes the BW distance (which they took from Storm et al.) equal to the independently known distance. They find $p=$ $(1.462 \pm 0.087)+(-0.172 \pm 0.086) \log P$, or, excluding the outlier FF Aql, $p=(1.447 \pm 0.070)+(-0.159 \pm 0.070) \log P$.

Figure 7 shows the distance to the LMC Cepheids versus $\log P$ for $p=1.33$. As the distance is proportional to the $p$-factor and since the dependence on period is assumed to be linear in $\log P$, the slope in this plot indicates what the $p-\log P$ dependence should be to have no dependence of distance on period. Depending on whether the slope is derived from the bisector (the dashed line), or a weighted least-squares fit (the solid line), the result is $-0.28 \pm 0.05$ or $-0.21 \pm 0.04$, respectively.

One can also make the consideration that the distance should be independent of (mean) $(V-K)$ colour. Figure 7 shows the result for $p=1.33$. As period and colour are related, this is not independent of the earlier estimate. Trying various coefficients, it is found that a slope of $-0.21 \pm 0.05$ or $-0.24 \pm 0.05$ will give no dependence on colour, for the bisector and weighted leastsquares fit, respectively.

The BW method is primarily of interest because it gives absolute distances. For the Cepheids in the LMC, one can also expect and demand that the slope of the PL relation in the $V$ - and $K$-band is independent of whether it is derived from $M_{\mathrm{V}}$ and $M_{K}$ taking the BW distances or from the purely observed mean $V_{0}$ and $K_{0}$ magnitudes.

The observed PL relations are listed in Table 9 and shown in Fig. 8. Apart from $V$ and $K$, the reddening free combination of these colours is also included, $W(V K)=K-0.13(V-K)$ (see Inno et al. 2012). Three solutions are listed, depending on whether the LMC Cepheids are put at the barycenter or not. In the former case, one corrects for the tilt and orientation of the LMC disk. The model by van der Marel \& Cioni (2001) was used (as Storm et al. 2011b did). It is based mainly on AGB stars located between 2.5 and $6.7^{\circ}$ from the LMC centre. An alternative model used is that by Nikolaev et al. (2004), which is based on 2100 Cepheids within $4^{\circ}$ from the centre. The slope and zero point of the observed PL relations are very similar, independent of the type of correction. The smallest dispersion in the $K$-band is actually achieved when applying no correction. The solution was calculated using a bisector and a weighted least-squares fit and is largely in agreement. The slopes derived here from the observations are in excellent agreement with literature values, some of which are also listed in Table 9.

As the absolute magnitudes depend on the distance, which in turn depends on the $p$-factor, changing the slope in the $p-\log P$ relation will change the slope of the PL relation. Demanding that the two are equal, it is found that the slope is $-0.25 \pm 0.05$ and $-0.25 \pm 0.05$, in the $K-$ and $V$-band, respectively. Taking the 
M. A. T. Groenewegen: Baade-Wesselink distances and the effect of metallicity in Cepheids
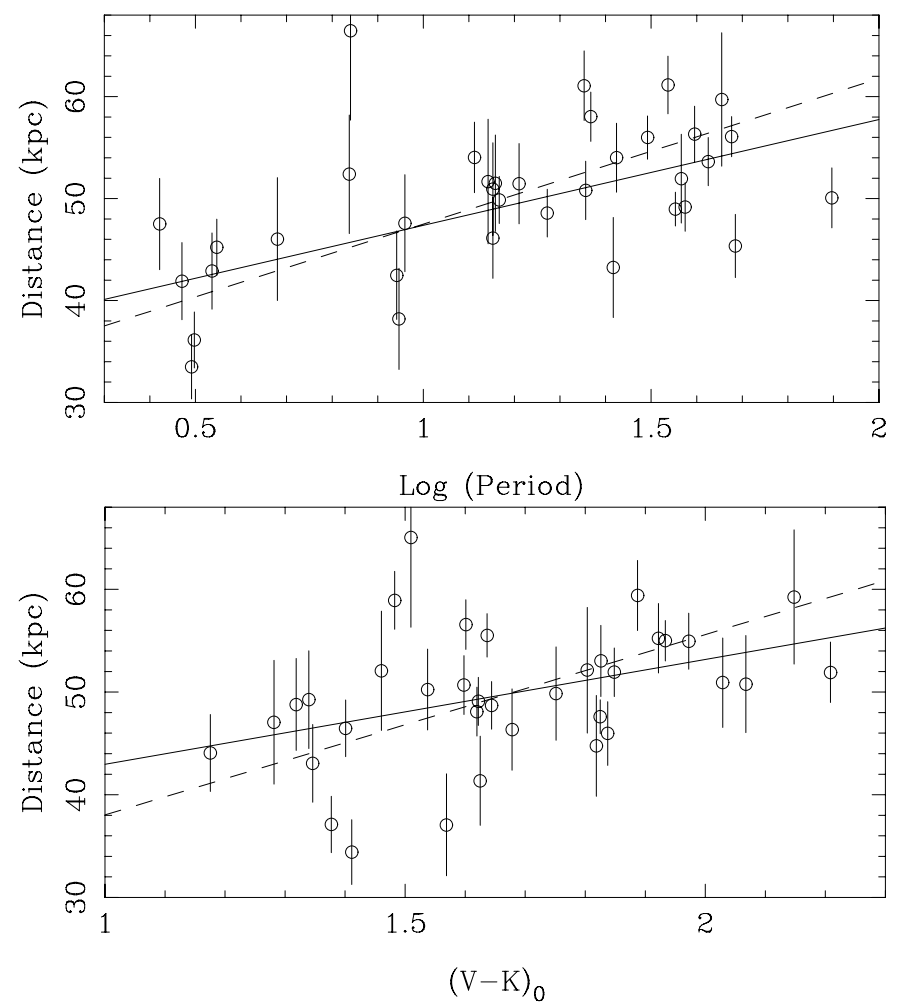

Fig. 7. Distance to the barycenter of the LMC against $\log P$ and against mean $(V-K)$ colour for a constant $p$-factor of 1.33. The dashed line indicates the bisector fit, the solid line the least-squares solution.

average of these six determinations, the finally adopted relation is $p=p_{o}-0.24 \log P$ (with an estimated error of 0.03 ). The dependence of distance on $\log P$ and $(V-K)$ colour for the LMC Cepheids is shown in Fig. 9.

\subsection{The zero point of the $p-\log P$ relation}

As in Storm et al. (2011a) the zero point of the $p-\log P$ is based on stars that have an independent distance, namely those with a parallax (the ten stars listed in Table 6) and the Cepheids that are in clusters. For the latter, Storm et al. (2011a,b) used the associations and distances in Turner (2010). However, this list is not complete (cf. Tammann et al. 2003), and some results have become available since 2010. A discussion of the distances adopted for Cepheids in clusters in the present sample is presented in Appendix A.

The relation finally adopted is $p=1.50-0.24 \log P$. For this relation, the weighted mean of the ratio of reference distance to BW distance (see later) is $1.000 \pm 0.026$ for eight stars which have a parallax (the two outliers that deviate by more than $3.4 \sigma$ and that are removed are W Sgr with a ratio of $2.20 \pm 0.36$, and FF Aql with $0.58 \pm 0.11$ ), and $0.999 \pm 0.014$ for the 18 stars in clusters. In the latter case, the three outliers that deviate by more than $4.5 \sigma$ and that are removed are SU Cyg with a ratio of $0.76 \pm 0.05$, VY Car with $1.57 \pm 0.11$, and X Cyg with $1.23 \pm$ 0.04 , while the recent determinations for $\delta \mathrm{Cep}$ and $\zeta$ Gem were already considered when deriving the best distances for the stars with parallaxes (see Table 6).

In comparison, Storm et al. (2011a) base their zero point solely on nine stars with HST parallax (W Sgr is excluded as well, but its BW distance for FF Aql agrees with the HST-based one). An aposteriori comparison to the Cepheids in clusters (with
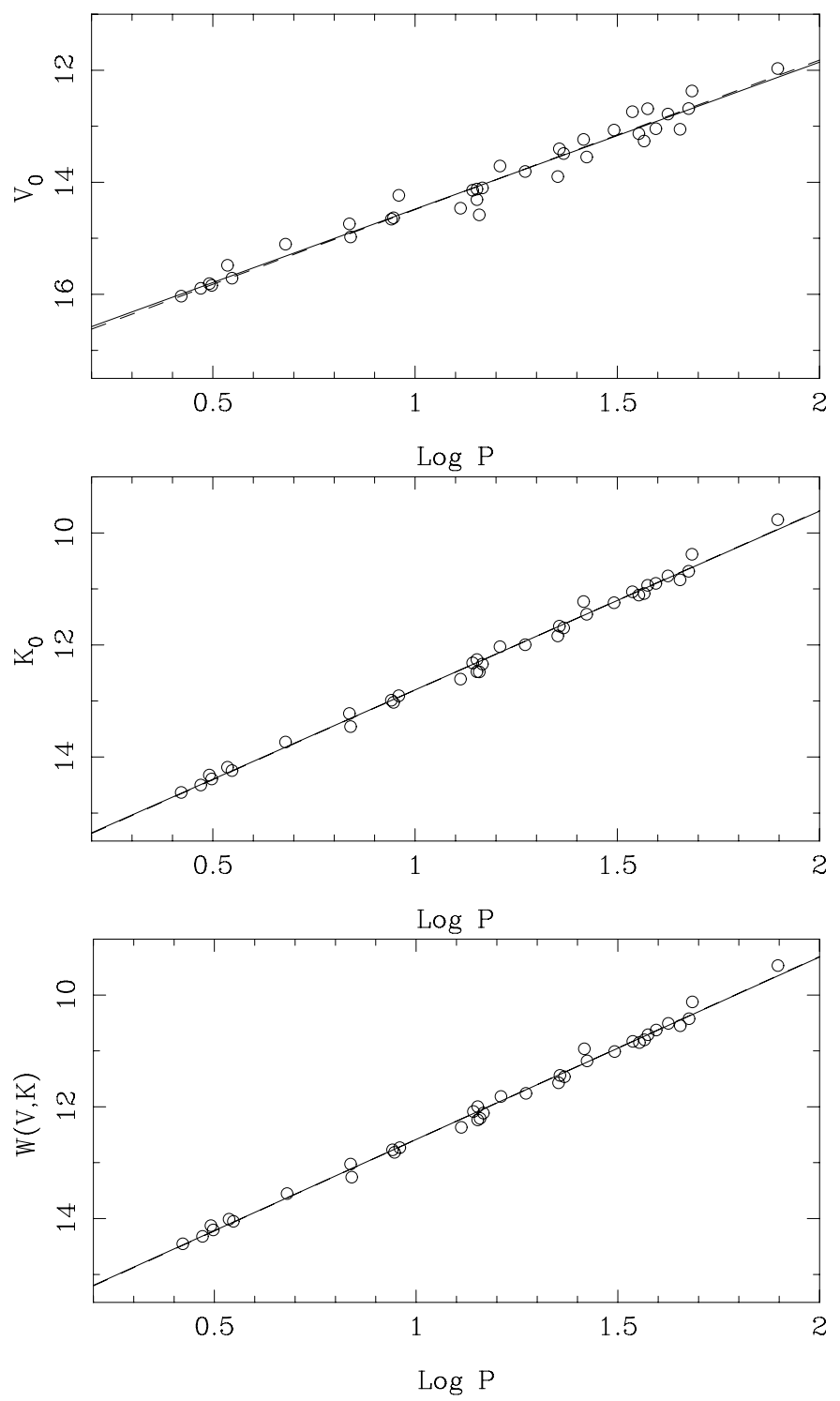

Fig. 8. Observed $V$-band (upper panel), $K$-band (middle) and $W(V K)$ (bottom) PL relations for the LMC Cepheids. The dashed line indicates the bisector fit, the solid line the least-squares solution (indistinguishable except in the upper panel).

the distances from Turner 2010) using 16 stars in common with their Galactic Cepheid sample (of which SU Cas is excluded, as the result from Majaess et al. (2012c) was not available to them) shows an unweighted mean difference of $0.12 \pm 0.06$ in distance modulus.

Table 10 lists the distances, radii, and absolute magnitudes obtained for the Galactic, LMC, and SMC Cepheids. The table also lists the adopted $E(B-V)$ and error bar, the derived period, and the $p$-factor following $p=1.50-0.24 \log P$ adopted in the present paper. The distances, radii, and errors scale directly with $p$. The error in the period is a few units in the last decimal place. For the derived quantities two error bars are quoted. For the distance and radius, the first error bar listed is the error in the fit, and for the absolute magnitudes the error is due to the error in distance and $E(B-V)$. The second error quoted is based on a Monte Carlo simulation, where (1) new datasets are generated based on the error bar in each individual $V, K$, and RV measurement, (2) the analysis takes into account an $E(B-V)$ value randomly drawn from a Gaussian distribution based on the listed 
Table 9. Observed, using a bisector and weighted least-squares fit, and literature PL relations in the LMC.

\begin{tabular}{lccc}
\hline \hline Relation & Bisector & wLSQ & Remarks \\
\hline Observed $K$-band & $16.01 \pm 0.05$ & $15.99 \pm 0.04$ & Correction van der Marel \& Cioni (2001) \\
& $-3.20 \pm 0.04$ & $-3.19 \pm 0.04$ & \\
Observed $K$-band & $16.03 \pm 0.05$ & $16.02 \pm 0.04$ & Correction Nikolaev et al. (2004) \\
& $-3.22 \pm 0.04$ & $-3.21 \pm 0.04$ & \\
Observed $K$-band & $16.06 \pm 0.04$ & $16.05 \pm 0.03$ & No correction \\
& $-3.25 \pm 0.03$ & $-3.24 \pm 0.03$ & \\
Literature $K$-band & & $15.996 \pm 0.010$ & Ngeow et al. (2009) \\
& & $-3.194 \pm 0.015$ & \\
Literature $K$-band & & $16.070 \pm 0.017$ & Ripepi et al. (2012) \\
& & $-3.295 \pm 0.018$ & \\
Observed $V$-band & $17.15 \pm 0.08$ & $17.09 \pm 0.07$ & Correction van der Marel \& Cioni (2001) \\
& $-2.67 \pm 0.07$ & $-2.62 \pm 0.07$ & \\
Observed $V$-band & $17.18 \pm 0.07$ & $17.13 \pm 0.07$ & Correction Nikolaev et al. (2004) \\
& $-2.69 \pm 0.06$ & $-2.65 \pm 0.06$ & \\
Observed $V$-band & $17.21 \pm 0.07$ & $17.16 \pm 0.07$ & No correction \\
& $-2.72 \pm 0.06$ & $-2.68 \pm 0.06$ & \\
Literature $V$-band & & $17.115 \pm 0.015$ & Ngeow et al. (2009) \\
& & $-2.769 \pm 0.023$ & \\
Literature $V$-band & & $-1.304 \pm 0.065$ & Turner et al. (2010) \\
& & $-2.786 \pm 0.075$ & \\
Observed $W(V K)$ & $15.86 \pm 0.05$ & $15.85 \pm 0.04$ & Correction van der Marel \& Cioni (2001) \\
& $-3.27 \pm 0.04$ & $-3.26 \pm 0.04$ & \\
Observed $W(V K)$ & $15.88 \pm 0.04$ & $15.87 \pm 0.04$ & Correction Nikolaev et al. (2004) \\
& $-3.30 \pm 0.04$ & $-3.29 \pm 0.04$ & \\
Observed $W(V K)$ & $15.91 \pm 0.03$ & $15.91 \pm 0.03$ & Inno et al. (2012) \\
Literature $W(V K)$ & $-3.32 \pm 0.03$ & $-3.32 \pm 0.03$ & \\
& & $15.901 \pm 0.005$ & \\
Literature $W(V K)$ & & $-3.326 \pm 0.008$ & \\
& & $15.870 \pm 0.013$ & \\
\hline
\end{tabular}

Notes. The zero point is listed in the first line, the slope in the second line.
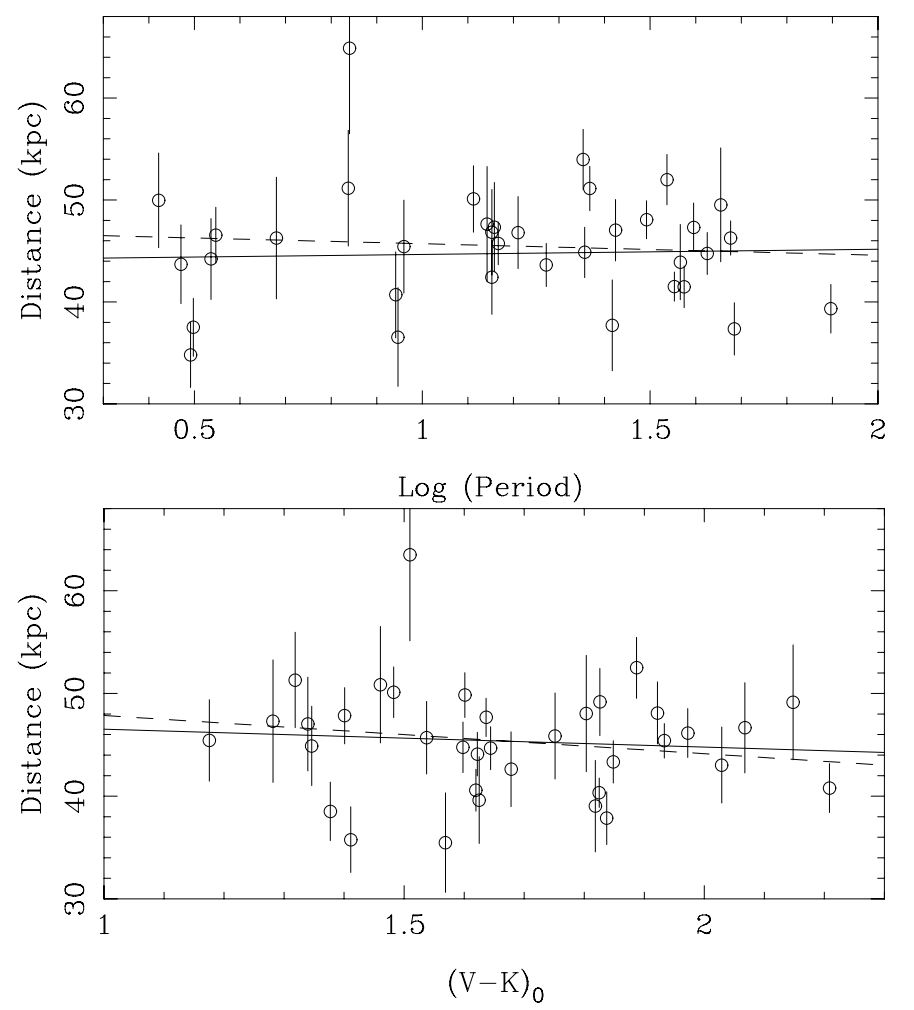

Fig. 9. Distance to the barycenter of the LMC against $\log P$ and against mean $(V-K)$ colour for a $p$-factor of $1.50-0.24 \log P$. The dashed line indicates the bisector fit, the solid line the least-squares solution. mean value and $1 \sigma$ error bar, (3) a random error in the $p$-factor of 0.02 units, and (4) a variation in the number of harmonics used to describe the optical, infrared, and RV curves. The second error quoted is the $1 \sigma$ dispersion in the derived quantities.

Figure 10 illustrates the fit to the $V, K$, and RV curve for AQ Pup, while Fig. 11 shows the variation of the angular diameter against phase and the change in angular diameter derived from the SB relation against the change in radius from integration of the RV curve from which the distance is derived (see Eq. (5)). Figures similar to Figs. 10 and 11 for all stars in the sample are available from the author.

\section{3. $P L(Z)$ relations}

Table 11 presents PL and PLZ relations of the form $M=$ $\alpha \log P+\beta+\gamma[\mathrm{Fe} / \mathrm{H}]$ in the $V, W(V K)$, and $K$-band. The results are given for the SMC, LMC, Galaxy, and for all Cepheids. Because of the small numbers of SMC Cepheids, the results for that galaxy are not reliable and are given for completeness only. For the Galactic Cepheids only and for the complete sample, a few clear outliers are removed (deviating by more than 0.8 mag from the PL relation).

Figures 12 and 13 show the PL relation in the $V$-band and $K$-band for the complete sample; in the bottom panel, the residual plotted against metallicity is shown. The first and second error bar quoted for the magnitudes in Table 10 have been added in quadrature.

The metallicity dependence was determined in two ways: 1) by first fitting a linear PL relation and fitting the residual with 
Table 11. $\mathrm{PL}(\mathrm{Z})$ relations of the form $M=\alpha+\beta \log P+\gamma[\mathrm{Fe} / \mathrm{H}]+\delta \log P[\mathrm{Fe} / \mathrm{H}]$, in the $V, W(V K)$, and $K$-band and for different galaxies.

\begin{tabular}{|c|c|c|c|c|c|c|}
\hline Band & Galaxy & $N$ & $\alpha$ & $\beta$ & $\gamma$ & $\delta$ \\
\hline $\bar{K}$ & ALL & 162 & $-2.50 \pm 0.08$ & $-3.06 \pm 0.06$ & - & \\
\hline$K$ & GAL & 121 & $-2.55 \pm 0.09$ & $-3.03 \pm 0.08$ & - & \\
\hline$K$ & LMC & 36 & $-2.26 \pm 0.17$ & $-3.21 \pm 0.13$ & - & \\
\hline$K$ & SMC & 6 & $-0.36 \pm 0.98$ & $-4.56 \pm 0.78$ & - & \\
\hline$K$ & ALL & 162 & $-2.49 \pm 0.08$ & $-3.07 \pm 0.07$ & $-0.05 \pm 0.10$ & \\
\hline$K$ & GAL & 121 & $-2.56 \pm 0.09$ & $-3.03 \pm 0.08$ & $0.07 \pm 0.20$ & \\
\hline$K$ & LMC & 36 & $-2.27 \pm 0.18$ & $-3.22 \pm 0.13$ & $0.19 \pm 0.37$ & \\
\hline$K$ & SMC & 6 & $-0.66 \pm 0.99$ & $-4.23 \pm 0.81$ & $-1.31 \pm 0.86$ & \\
\hline$K$ & ALL & 162 & $-2.47 \pm 0.08$ & $-3.08 \pm 0.07$ & $-0.59 \pm 0.42$ & $0.42 \pm 0.31$ \\
\hline$V$ & ALL & 160 & $-1.48 \pm 0.08$ & $-2.40 \pm 0.07$ & - & \\
\hline$V$ & GAL & 119 & $-1.68 \pm 0.10$ & $-2.21 \pm 0.09$ & - & \\
\hline V & LMC & 36 & $-1.10 \pm 0.17$ & $-2.69 \pm 0.12$ & - & \\
\hline$V$ & SMC & 6 & $0.73 \pm 0.93$ & $-4.03 \pm 0.74$ & - & \\
\hline$V$ & ALL & 160 & $-1.55 \pm 0.09$ & $-2.33 \pm 0.07$ & $0.23 \pm 0.11$ & \\
\hline$V$ & GAL & 121 & $-1.69 \pm 0.10$ & $-2.21 \pm 0.09$ & $0.17 \pm 0.25$ & \\
\hline$V$ & LMC & 36 & $-1.09 \pm 0.17$ & $-2.68 \pm 0.12$ & $-0.14 \pm 0.35$ & \\
\hline$V$ & SMC & 6 & $0.48 \pm 0.95$ & $-3.74 \pm 0.77$ & $-1.19 \pm 0.82$ & \\
\hline$V$ & ALL & 160 & $-1.54 \pm 0.09$ & $-2.34 \pm 0.08$ & $-0.04 \pm 0.46$ & $0.21 \pm 0.34$ \\
\hline$W V K$ & ALL & 158 & $-2.68 \pm 0.08$ & $-3.12 \pm 0.06$ & - & \\
\hline$W V K$ & GAL & 120 & $-2.69 \pm 0.09$ & $-3.12 \pm 0.08$ & - & \\
\hline$W V K$ & LMC & 36 & $-2.41 \pm 0.18$ & $-3.27 \pm 0.13$ & - & \\
\hline$W V K$ & SMC & 6 & $-0.51 \pm 0.98$ & $-4.63 \pm 0.78$ & - & \\
\hline$W V K$ & ALL & 158 & $-2.69 \pm 0.08$ & $-3.11 \pm 0.07$ & $0.04 \pm 0.10$ & \\
\hline$W V K$ & GAL & 120 & $-2.72 \pm 0.09$ & $-3.13 \pm 0.08$ & $0.34 \pm 0.20$ & \\
\hline$W V K$ & LMC & 36 & $-2.42 \pm 0.18$ & $-3.29 \pm 0.13$ & $+0.23 \pm 0.37$ & \\
\hline$W V K$ & SMC & 6 & $-0.81 \pm 0.99$ & $-4.29 \pm 0.81$ & $-1.34 \pm 0.85$ & \\
\hline$W V K$ & ALL & 158 & $-2.70 \pm 0.09$ & $-3.11 \pm 0.07$ & $+0.17 \pm 0.44$ & $-0.10 \pm 0.33$ \\
\hline
\end{tabular}

a linear relation against $[\mathrm{Fe} / \mathrm{H}]$ (as shown in Figs. 12 and 13 by the solid line, done mainly for easy visualisation); and 2) by making a linear fit in the two variables $\log P$ and $[\mathrm{Fe} / \mathrm{H}]$, as listed in Table 11 and shown in Figs. 12 and 13 by the dashed line.

The slopes of the LMC $(-3.21 \pm 0.13)$ and Galactic $(-3.03 \pm$ $0.08) \mathrm{PL}$ relation in the $K$-band are formally consistent at the $1 \sigma$ level; in the $V$-band they are consistent only at the $3 \sigma$ level $(-2.69 \pm 0.12)$ for the LMC, $(-2.21 \pm 0.09)$ for the Galaxy). The better agreement in $K$ and lesser agreement in $V$ between the two galaxies is in line with theoretically predicted relations (Bono et al. 2010), which give slopes at $Z=0.004$, $0.008,0.02$ in the $K$-band of $-3.19 \pm 0.09,-3.28 \pm 0.09$, and $-3.22 \pm 0.15$, respectively, (and $\delta$ slope $/ \delta \log Z=0.08 \pm 0.07$ over all metallicities they considered), and in the $V$-band of $-2.87 \pm 0.09,-2.80 \pm 0.15$, and $-2.51 \pm 0.24$, respectively (and $\delta$ slope $/ \delta \log Z=0.67 \pm 0.09)$. The slope found for the Galactic Cepheids in the $V$-band $(-2.21 \pm 0.09)$ agrees at the $1.5 \sigma$ level with the value of $(-2.43 \pm 0.12)$ in Benedict et al. (2007) based on the ten stars with HST parallaxes. In the $K$-band, the slope $(-3.03 \pm 0.08)$ agrees at the $2 \sigma$ level with the $(-3.32 \pm 0.12)$ found by Benedict et al. The slopes found for the LMC Cepheids in the $V$-band $(-2.69 \pm 0.12)$ and $K$-band $(-3.21 \pm 0.13)$ are in excellent agreement with various determinations in the literature, see Sect. 6.1 and Table 9.

The metallicity dependence quoted in G08 based on 68 Galactic Cepheids were $\gamma=(+0.27 \pm 0.30) \mathrm{mag} / \mathrm{dex}(V$-band $)$ and $(-0.11 \pm 0.24)(K$-band $)$. Now, values of $(+0.17 \pm 0.25)$ and $(+0.07 \pm 0.20)$ from 121 Galactic Cepheids and $(+0.23 \pm 0.11)$ and $(-0.05 \pm 0.10) \mathrm{mag} / \mathrm{dex}$ from the complete sample are derived. Compared to G08, the larger sample of Galactic Cepheids has reduced the error bar, but the main reduction in error bar has come from adding the MC Cepheids. The outcome is that the iron dependence of the PL relation in the $K$-band is not significant and only marginally significant $(2 \sigma)$ in the $V$ band.
A fit were the slope of the PL relation is also allowed to vary linearly with metallicity is also included in Table 11 but the error bars in the coefficients are large and the result is not significant.

\subsection{The PR relation}

Figure 14 shows the PR relation for all Galactic and MC Cepheids. The best fit is

$\log R=(0.651 \pm 0.012) \log P+(1.136 \pm 0.014), \sigma=0.055$,

where 8 outliers (deviating by 0.17 dex, or about $3 \sigma$ ) have been removed $^{6}$.

This result does depend on the adopted $p$-factor relation. For a constant $p=1.33$, for example, the relation would become $\log R=0.737 \log P+1.074$. This is in agreement with Molinari et al. (2011), who find $\log R=(0.75 \pm 0.03) \log P+(1.10 \pm$ 0.03 ) for a constant $p=1.27$. A negative dependence of the $p$-factor on period leads to a shallower slope. The PR relation in Eq. (7) based on stars with known distance has a slope of $0.696 \pm 0.033$. This is in agreement with the present one, which depends on the $p$-factor. The slopes in the PR relation that are found in the present work are shallower than often quoted in the literature (see Molinari et al. (Molinari2011), and Turner et al. (2010) for recent compilations), but these also depend on the $p$-factor. For example, Gieren et al. (1998) find $\log R=(0.750 \pm$ $0.024) \log P+(1.075 \pm 0.007)$ for $p=1.39-0.03 \log P$, while Turner \& Burke (2002) find $\log R=(0.750 \pm 0.006) \log P+$ $(1.071 \pm 0.006)$ for $p=1.31$.

Theory predicts slopes shallower than this and more in agreement with the slopes found in the present paper. Recent models by Petroni et al. (2003) lead to $\log R=(0.676 \pm 0.006)$

\footnotetext{
6 These are AN Aur, CR Ser, DT Cyg, V495 Cyg, VX Per, VY Cyg, W Sgr, and HV 1335.
} 

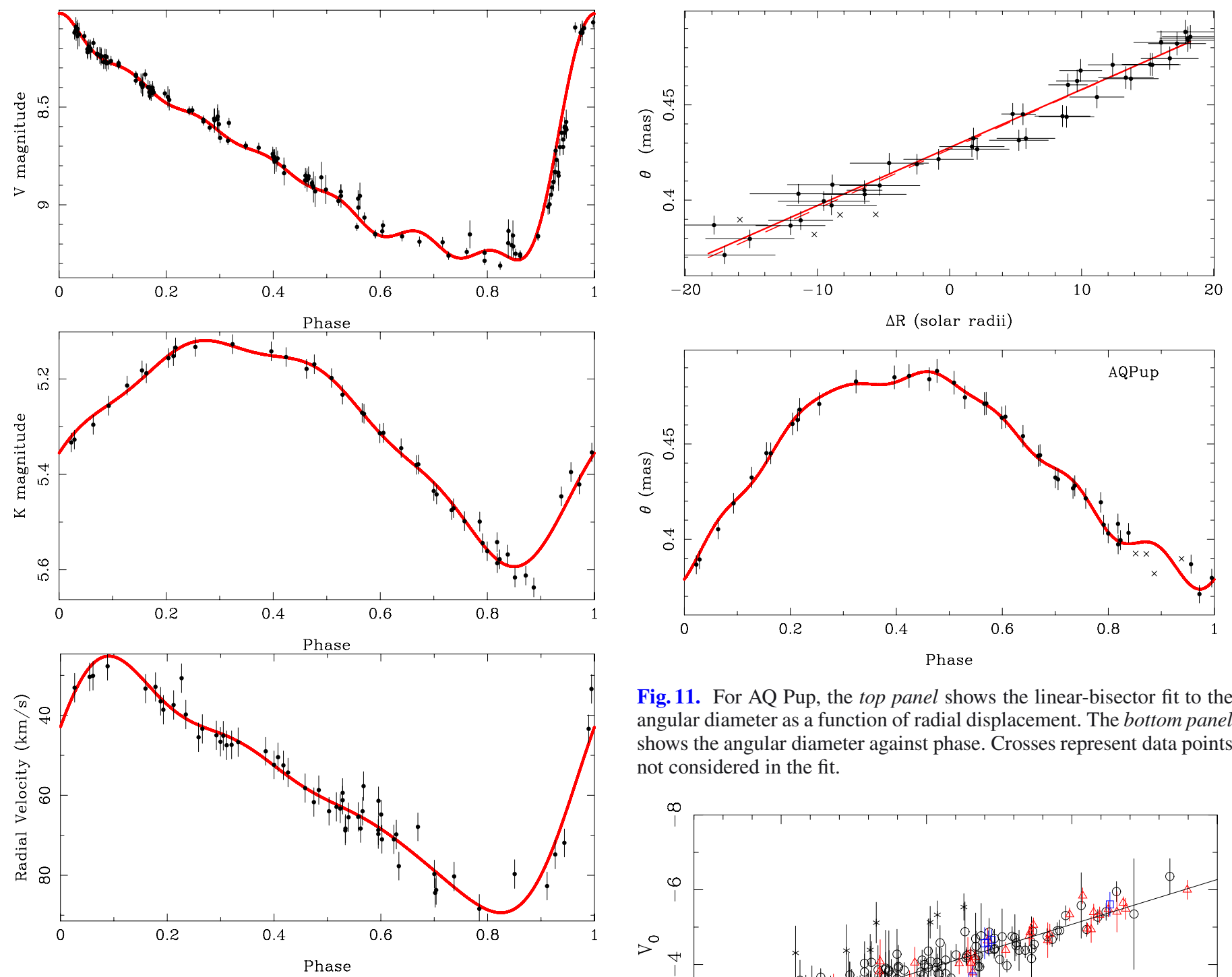

Fig. 10. Phased curves in $V, K$, and RV are shown for AQ Pup. Data points are shown with errors bars and the line shows the harmonic fit.

$\log P+(1.173 \pm 0.008)$ for solar-metallicity. Both theory and radii for stars with known distances lead to slopes in the PR relation shallower than found for BW-type analysis with a constant $p$-factor and thus support a (steep) period dependence of the $p$-factor.

\section{Summary and discussion}

The PL relation in the $V$ and $K$-band (and the corresponding Wesenheit index) and the dependence on metallicity are investigated for a sample of 128 Galactic, $6 \mathrm{SMC}$, and 36 LMC Cepheids with an individual metallicity determination from high-resolution spectroscopy. Distances are derived using the Baade-Wesselink technique implementing the most recent surface-brightness relation and estimates for the projection factor.

The slope of the $p$-factor relation is found to be -0.24 from demanding that the distance to the LMC does not depend on period and $(V-K)$ colour and that the slope of the PL relation in $V$ and $K$ be the same for the observed relations based on apparent and absolute magnitudes. This result agrees within the errors with the slope found by Storm et al. (2011a). The slope found

Fig. 11. For AQ Pup, the top panel shows the linear-bisector fit to the angular diameter as a function of radial displacement. The bottom panel shows the angular diameter against phase. Crosses represent data points not considered in the fit.
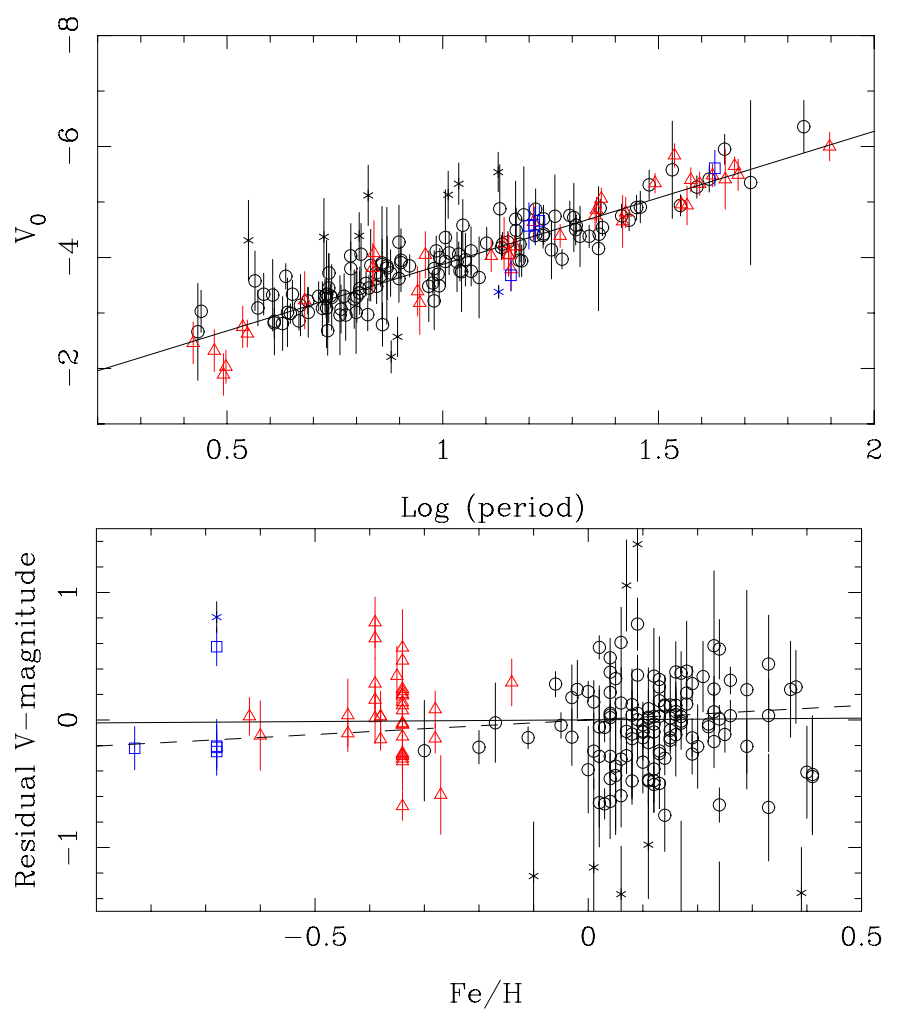

Fig. 12. The PL relation in the $V$-band. Galactic objects are plotted as (black) open circles, LMC Cepheids as (red) triangles, and SMC Cepheids as (blue) squares. Stars plotted with a cross symbol are excluded from the fit. The bottom panel shows the residual plotted versus metallicity. The solid line shows the fit when the residual is fitted, the solid line the dependence from a two-parameter fit (as given in Table 11). 

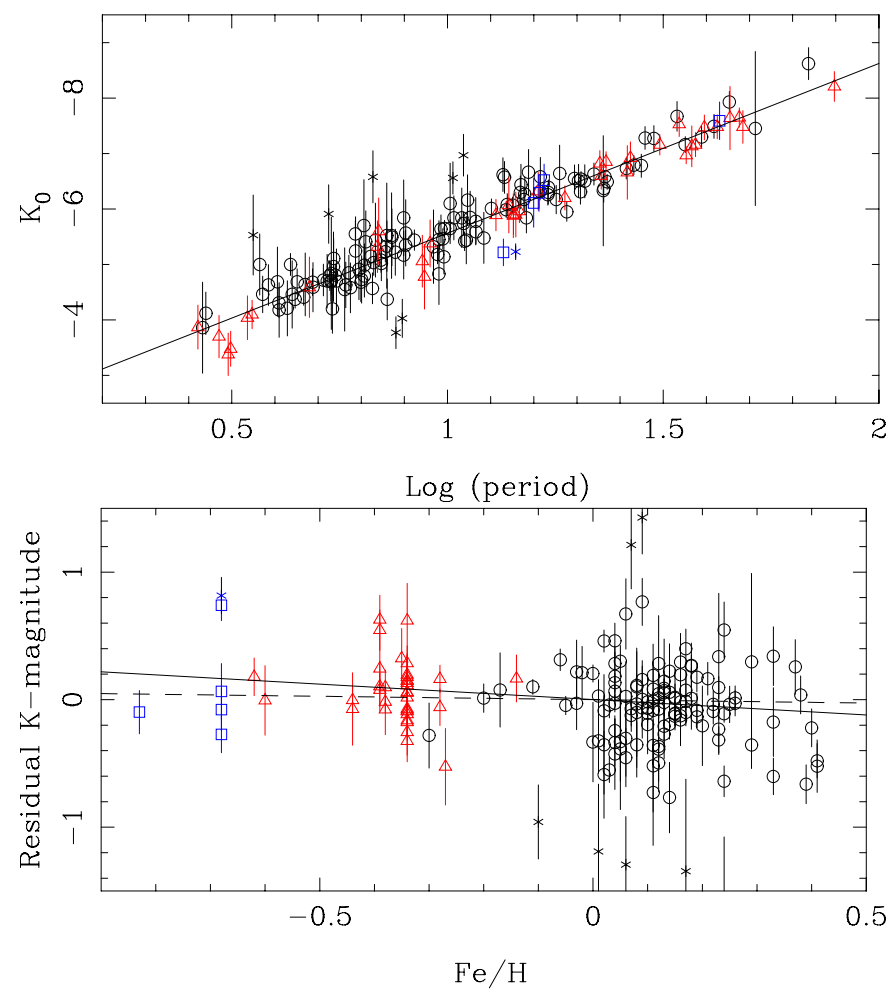

Fig. 13. As Fig. 12 in the $K$-band.

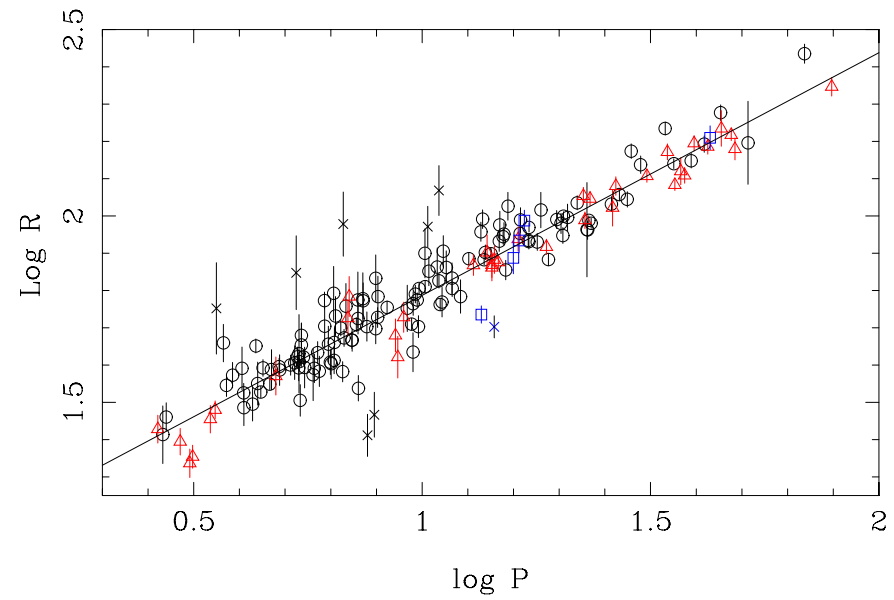

Fig. 14. The PR relation the all Cepheids. Galactic objects are plotted as (black) open circles, LMC Cepheids as (red) triangles, and SMC Cepheids as (blue) squares. Stars plotted with a cross symbol are excluded from the fit. The line is the best fit listed in Eq. (10).

by Storm et al. and in the present work is much steeper than the one predicted by theory, $p=(-0.08 \pm 0.05) \log P+(1.31 \pm 0.06)$ (Nardetto et al. 2009).

The zero point of the relation is tight to the ten Cepheids that have HST and improved HIPPARCos parallaxes (and a cluster distance for two Cepheids) and to the 18 Cepheids that have only a cluster distance. The finally adopted relation is $p=1.50-0.24 \log P$.

The metallicity dependence of the PL relation is investigated. No significant dependence is found in $K$, and $W(V K)$, and a $2 \sigma$ result in $V, \gamma=+0.23 \pm 0.11$.

The distance scale found here is shorter than that in Storm et al. (2011a,b) because of the smaller $p$-factor found here. At a typical period of ten days, $(1.55-0.186 \log P) /(1.50-0.24 \log P)=1.364 / 1.26=1.08$. This is reflected in the distance to the MCs. The median distance to the $36 \mathrm{LMC}$ and $6 \mathrm{SMC}$ Cepheids is 45.5 and $55.7 \mathrm{kpc}$ (Distance Modulus (DM) of 18.29 and 18.73). The error in the mean is 0.47 and $1.4 \mathrm{kpc}(0.02$ and 0.06 in DM).

Although the absolute distances are short for the adopted $p$-factor, the difference in DM between SMC and LMC is 0.44 \pm 0.06 , which is in agreement with other independent determinations (Type II Cepheids, red clump, tip of the RGB), see the discussion in Matsunaga et al. (2011). Storm et al. (2011b) found $0.47 \pm 0.14$.

The difference with respect to Storm et al. in the distance scale is unlikely to be related to reddening. Their values for $E(B-V)$ have been adopted for the MC Cepheids. For the 74 Galactic Cepheids in common, the average difference in adopted $E(B-V)$ is only 0.002 .

In general, however, changes in reddening do influence the derived distances. For example, the present Galactic sample has 50 stars in common with the sample in Pejcha \& Kochanek (2012). The average difference in $E(B-V)$ is -0.023 (theirs minus the present work), while for 21 LMC and 3 SMC stars in common with Pejcha \& Kochanek, the difference is +0.034 and +0.030 .

As a test the distances were re-computed, changing all $E(B-V)$ by +0.05 for the MC Cepheids and by -0.03 for the Galactic ones. The average LMC (SMC) distance is increased by $+640(+900)$ pc, while the distance to the Cepheids with parallaxes and in clusters is decreased by on average $-0.85 \%$. In other words, systematic effects in $E(B-V)$ of this magnitude would increase the DM to LMC and SMC to 18.34 and 18.78, respectively.

The exact procedure of comparing BW to HST-based distances also plays a role. Here, the weighted mean of the ratio has been used. Storm et al. use the unweighted mean of the difference in distances relative to the average of the two distances. If this procedure is adopted then a distance scale longer by $3.7 \%$ is found (corresponding to DM of 18.37 and 18.81, respectively).

The distance to the LMC is shorter than most of the recent determinations, which are in the range 18.42-18.55 (Walker 2012 , who quotes $49.7 \mathrm{kpc}$ with a range of $\pm 3 \%$ ). Within the current framework, this difference could be reconciled by suggesting that the $p$-factor is larger at lower metallicity by about $\sim 8-9 \%$ at the metallicity of both the LMC and SMC. This is not predicted by theory (Nardetto et al. 2009), but then theory currently also does not predict the steep dependence of the $p$-factor on period either.

Further improvements could still be made on the observational side. Metallicity determination from high-resolution spectroscopy for the $5 \mathrm{SMC}$ and (a significant subset of the) $22 \mathrm{LMC}$ Cepheids in the sample without such determination would likely further improve the determination of the metallicity term in the PL relation. Interferometry for more stars could improve on better constraining the surface-brightness relation, possibly further investigating the hint of a metallicity dependence noted in Sect. 4. The observations of T Vul (Gallenne et al. 2012) demonstrate that angular diameters as small as $0.60^{\prime \prime}$ can be determined reliably in the $K$-band on $300 \mathrm{~m}$ baselines.

For reference, Table 12 lists the stars with minimum angular diameters $\geq 0.59^{\prime \prime}$ and angular diameter amplitude $\geq 0.06^{\prime \prime}$, sorted by the latter quantity. It includes objects for which interferometric observations already exist (including objects that would benefit from additional observations, like X Sgr and W Sgr). 
Table 12. Galactic Cepheids with the largest angular diameters, sorted by amplitude.

\begin{tabular}{lccc}
\hline \hline Name & $\begin{array}{c}\theta_{\min } \\
\left({ }^{\prime}\right)\end{array}$ & $\begin{array}{c}\theta_{\max }-\theta_{\min } \\
\left({ }^{\prime \prime}\right)\end{array}$ & {$[\mathrm{Fe} / \mathrm{H}]$} \\
\hline T Vul & 0.59 & 0.06 & 0.08 \\
RT Aur & 0.65 & 0.07 & 0.13 \\
AW Per & 0.62 & 0.08 & 0.04 \\
S Mus & 0.67 & 0.08 & 0.07 \\
S Nor & 0.64 & 0.08 & 0.13 \\
W Gem & 0.62 & 0.08 & 0.02 \\
RX Cam & 0.68 & 0.09 & 0.11 \\
S Sge & 0.73 & 0.09 & 0.15 \\
U Sgr & 0.70 & 0.09 & 0.15 \\
U Vul & 0.71 & 0.09 & 0.19 \\
GY Sge & 0.61 & 0.11 & 0.29 \\
S Vul & 0.60 & 0.11 & 0.12 \\
U Aql & 0.70 & 0.11 & 0.17 \\
Y Sgr & 0.79 & 0.11 & 0.12 \\
Y Oph* & 1.36 & 0.12 & 0.13 \\
RY Sco & 0.68 & 0.13 & 0.16 \\
X Sgr* & 1.41 & 0.14 & -0.20 \\
TT Aql & 0.65 & 0.16 & 0.22 \\
zeta Gem* & 1.60 & 0.16 & 0.10 \\
del Cep* & 1.38 & 0.17 & 0.12 \\
RZ Vel & 0.59 & 0.17 & 0.04 \\
X Cyg & 0.70 & 0.18 & 0.17 \\
SV Vul & 0.71 & 0.20 & 0.12 \\
beta Dor* & 1.71 & 0.21 & 0.10 \\
eta Aql* & 1.65 & 0.21 & 0.15 \\
U Car & 0.86 & 0.22 & 0.04 \\
RS Pup & 0.78 & 0.24 & 0.22 \\
T Mon & 0.80 & 0.24 & 0.23 \\
W Sgr* & 1.11 & 0.25 & 0.09 \\
l Car* & 2.64 & 0.59 & 0.13 \\
\hline
\end{tabular}

Notes. ${ }^{(*)}$ A star that has been monitored interferometrically, see Table 6.

Acknowledgements. This research has made use of the SIMBAD database, operated at CDS, Strasbourg, France. M.G. would like to thank Alexandre Gallenne for providing the individual angular-diameter determinations for $\mathrm{T}$ Vul and FF Aql, Giuseppe Bono and Laura Inno for providing their latest results on MC PL relations before publication, and Giuseppe, Jesper Storm and Chow Choong Ngeow for commenting on a earlier version of the paper.

\section{References}

Abt, H. A. 1959, AJ, 130, 1021

Abt, H. A. 1973, ApJS, 26, 365

Abt, H. A., \& Levy, S. G. 1978, PASP, 90, 188

Adams, W. S., \& Shapley, H. 1918, ApJ, 47, 46

Akritas, M. G., \& Bershady, M. A. 1996, ApJ, 470, 706

Albrow, M. D., \& Cottrell, P. L. 1996, MNRAS, 280, 917

Alcock, C., Allsman, R. A., Axelrod, T. S., Bennett, D. P., \& Cook, K. H. 1995, AJ, 109, 1653

An, D., Terndrup, D. M., \& Pinsonneault, M. H. 2007, ApJ, 671, 1640

Andrievsky, S. M., Kovtyukh, V. V., Luck, R. E., et al. 2002, A\&A, 381, 32

Andrievsky, S. M., Egorova, I. A., Korotin, S. A., \& Kovtyukh, V. V. 2003, AN, 324,532

Arp, H., \& Thackeray, A. D. 1967, ApJ, 149, 73

Babel, J., Burki, G., Mayor, M., Chmielewski, Y., \& Waelkens, C. 1989, A\&A, 216, 125

Barnes, T. G., Moffett, T. J., \& Slovak, M. H. 1987, ApJS, 65, 307

Barnes, T. G., Moffett, T. J., \& Slovak, M. H. 1988, ApJS, 66, 43

Barnes, T. G., Fernley, J. A., Frueh, M. L., et al. 1997, PASP, 109, 645

Barnes, T. G., Jeffery, E. J., Berger, J. O., et al. 2003, ApJ, 592, 539

Barnes, T. G., Jeffery, E. J., Montemayor, T. J., \& Skillen, I. 2005a, ApJS, 156, 227

Barnes, T. G., Storm, J., Jefferys, W. J., Gieren, W. P., \& Fouqué, P. 2005b, ApJ, 631,572
Beavers, W. I., \& Eitter, J. J. 1986, ApJS, 62, 147

Benedict, G. F., McArthur, B. E., Feast, M. W., et al. 2007, AJ, 133, 1810

Berdnikov, L. N., Dambis, A. K., \& Vozyakovs, O. V. 2000, A\&AS, 143, 211

Bersier, D. 2002, ApJS, 140, 465

Bersier, D., Burki, G., Mayor, M., \& Duquennoy, A. 1994, A\&AS, 108, 25

Böhm-Vitense, E., Clark, M., Cottrell, P. L., \& Wallerstein, G. 1990, AJ, 99, 353

Bono, G., Caputo, F., Marconi, M., \& Musella, I. 2010, ApJ, 715, 277

Breger, M. 1967, MNRAS, 136, 61

Breger, M. 1970, AJ, 75, 239

Breitfellner, M. G., \& Gillet, D. 1993, A\&A, 277, 541

Caldwell, J. A. R., Coulson, I. M., Spencer Jones, J. H., Black, C. A., \& Feast, M. W. 1986, MNRAS, 220, 671

Caldwell, J. A. R., Coulson, I. M., Dean, J. F., \& Berdnikov, L. N. 2001, JAR, 7, 4

Campbell, W. W., \& Moore, J. H. 1928, Lick Obs. Bull., 16, 267

Carter, B. S. 1990, MNRAS, 242, 1

Coulson, I. M. 1983, MNRAS, 203, 925

Coulson, I. M., \& Caldwell, J. A. R. 1985a, SAAO Circulars, 9, 5

Coulson, I. M., \& Caldwell, J. A. R. 1985b, MNRAS, 216, 671

Coulson, I. M., Caldwell, J. A. R., \& Gieren, W. P. 1985, ApJS, 57, 595

Davis, J., Jacob, A. P., Robertson, J. G., et al. 2009, MNRAS, 394, 1620

Dean, J. F. 1977, MNSSA, 36, 3

Dean, J. F. 1981, SAAOC, 6, 10

Duncan, J. C. 1908, Lick Obs. Bull., 5, 82

Duncan, J. C. 1922 , ApJ, 56, 340

Duncan, J. C. 1932, PASP, 44, 324

Eggen, O. J. 1977, ApJS, 34, 33

Eggen, O. J. 1983, AJ, 88, 379

Evans, N. R. 1976, ApJS, 32, 399

Evans, N. R. 1983, ApJ, 272, 214

Evans, N. R. 1988, ApJS, 66, 343

Evans, N. R., \& Lyons, R. 1986, AJ, 92, 436

Evans, N. R., \& Lyons, R. 1994, AJ, 107, 2164

Evans, N. R., \& Sugars, B. J. A. 1997, AJ, 113, 792

Evans, N. R., \& Welch, D. L. 1993, PASP, 105, 836

Evans, N. R., Welch, D. L., Scarfe, C. D., \& Teays, T. J. 1990, AJ, 99, 1598

Evans, N. R., Welch, D. L., Slovak, M. H., Barnes, T. G. III, \& Moffett, T. J. 1993, AJ, 106, 1599

Evans, N. R., Carpenter, K., Robinson, R., et al. 1999, ApJ, 524, 379

Evans, N. R., Vinkó, J., \& Wahlgren, G. M. 2000, AJ, 120, 407

Evans, N. R., Berdnikov, L., Gorynya, N., Rastorguev, A., \& Eaton, J. 2011, AJ, 142,87

Feast, M. W. 1967, MNRAS, 136, 141

Feast, M. W. 1999, PASP, 111, 775

Feast, M. W., \& Walker, A. R. 1987, ARA\&A, 25, 345

Feast, M. W., Laney, C. D., Kinman, T. D., van Leeuwen, F., \& Whitelock, P. A. 2008, MNRAS, 386, 2115

Fernley, J. A., Skillen, I., \& Jameson, R. F. 1989, MNRAS 237, 947

Fouqué, P., \& Gieren, W. P. 1997, A\&A, 320, 799

Fouqué, P., Storm, J., \& Gieren, W. P. 2003, in Stellar candles for the extragalactic distance scale, Lect. Notes Phys., 635, 21

Fouqué, P., Arriagada, P., Storm, J., et al. 2007, A\&A, 476, 73 (F07)

Freedman, W. L., Grieve, G. R., \& Madore, B. F. 1985, ApJS, 59, 311

Frost, E. B. 1906, ApJ, 23, 264

Fry, A. M., \& Carney, B. W. 1997, AJ, 113, 1073

Gallenne, A., Kervella, P., Mérand, A., et al. 2012, A\&A, 541, A87

Gieren, W. P. 1976, A\&A 47, 211

Gieren, W. P. 1981a, ApJS, 46, 287

Gieren, W. P. 1981b, ApJS, 47, 315

Gieren, W. P. 1985, ApJ, 295, 507

Gieren, W. P. 1989a, A\&A, 216, 135

Gieren, W. P. 1989b, PASP, 101, 160

Gieren, W. P., Barnes T. G., \& Moffett, T. J. 1993, ApJ, 418, 135

Gieren, W. P., Welch, D. L., Mermilliod, J.-C., Matthews, J. M., \& Hertling, G. 1994, AJ, 107, 2093

Gieren, W. P., Fouqué, P., \& Gómez, M. 1997, ApJ, 488, 74

Gieren, W. P., Fouqué, P., \& Gómez, M. 1998, ApJ, 496, 17

Gieren, W. P., Gómez, M., Storm, J., et al., 2000, ApJS, 129, 111

Gieren, W. P., Storm, J., Barnes, T. G., et al. 2005, ApJ, 627, 224

Glass, I. S. 1985, IrAJ, 17, 1

Gorynya, N. A., Samus, N. N., Berdnikov, L. N., Rastorgouev, A. S., \& Sachkov, M. E. 1995, IBVS, 4199

Gorynya, N. A., Samus, N. N., Sachkov, M. E., et al. 1998, PAZh, 24, 939 (VizieR On-line Data Catalog: III/229)

Gould, A. 1994, ApJ, 426, 542

Grayzeck, E. J. 1978, AJ, 83, 1397

Groenewegen, M. A. T. 2007, A\&A, 474, 975 (G07)

Groenewegen, M. A. T. 2008, A\&A, 488, 25 (G08) 
Groenewegen, M. A. T., Romaniello, M., Primas, F., \& Mottini, M. 2004, A\&A, 420,655

Harper, W. E. 1934, Publ. DAO, 6, 151

Harris, H. C. 1980, Ph.D. Thesis, University of Washington

Häupl, W. 1988, AN, 309, 3

Henden, A. A. 1980, MNRAS, 192, 621

Herbig, G. H., \& Moore, J. H. 1952, ApJ, 116, 348

Hoyle, F., Shanks, T., \& Tanvir, N. R. 2003, MNRAS, 345, 269

Imbert, M. 1985, A\&AS, 58, 529

Imbert, M. 1989, A\&AS, 81, 339

Imbert, M. 1996, A\&AS, 116, 497

Imbert, M. 1999, A\&AS, 140, 79

Inno, L., Matsunaga, N., Bono, G., et al. 2012 [arXiv: 1212 .4376]

Isobe, T., Feigelson, E. D., Akritas, M. G., \& Babu, G. J. 1990, ApJ, 364, 104

Jacob, A. Ph. 2008, Ph.D. Thesis, University of Sydney

Jacobsen, T. S. 1970, ApJ, 159, 569

Jacobsen, T. S. 1974, ApJ, 191, 691

Jacobsen, T. S., \& Wallerstein, G. 1981, PASP, 93, 481

Joy, H. A. 1937, ApJ, 86, 363

Joy, H. A. 1952, ApJ, 115, 25

Kennicutt, R. C., Stetson, P. B., Saha, A., et al. 1998, ApJ, 498, 181

Kervella, P., Bersier, D., Mourard D., et al. 2004a, A\&A, 428, 587

Kervella, P., Fouqué, P., Storm, J., et al. 2004b, ApJ, 604, L113

Kervella, P., Nardetto, N., Bersier, D., Mourard, D., \& Coudé du Foresto, V. 2004c, A\&A, 416, 941

Kienzle, F., Moskalik, P., Bersier, D., \& Pont, F. 1999, A\&A, 341, 818

Kimeswenger, S., Lederle, C., Richichi, A., et al. 2004, A\&A, 413, 1037

Kiss, L. L. 1998, MNRAS, 297, 825

Kiss, L. L. 2000, MNRAS, 314, 420

Klagyivik, P., \& Szabados, L. 2009, A\&A, 504, 959

Kochanek, C. S. 1997, ApJ, 491, 13

Koen, C., Marang, F., Kilkenny, D., \& Jacobs, C. 2007, MNRAS, 380, 1433

Kovtyukh, V. V., Wallerstein, G., \& Andrievsky, S. M. 2005a, PASP, 117, 1173

Kovtyukh, V. V., Andrievsky, S.M., Belick, S. I., \& Luck, R. E. 2005b, AJ, 129, 433

Laney, C. D., \& Caldwell, J. A. R. 2007, MNRAS, 377, 147

Laney, C. D., \& Stobie, R. S. 1986, SAAO Circ, 10, 51

Laney, C. D., \& Stobie, R. S. 1992, A\&AS, 93, 93

Lemasle, B., Francois, P., Bono, G., et al. 2007, A\&A, 467, 283

Lenz, P., \& Breger, M. 2005, CoAst, 146, 53

Lloyd Evans, T. 1968, MNRAS, 141, 109

Lloyd Evans, T. 1980a, SAAO Circulars, 1, 163

Lloyd Evans, T. 1980b, SAAO Circulars, 1, 257

Luck, R. E., \& Andrievsky, S. M. 2004, AJ, 128, 343

Luck, R. E., \& Lambert, D. L. 2011, AJ, 142, 136

Luck, R. E., Gieren, W. P., Andrievsky, S. M., et al. 2003, A\&A, 401, 939

Luck, R. E., Kovtyukh, V. V., \& Andrievsky, S. M. 2006, AJ, 132, 902

Luck, R. E., Andrievsky, S. M., Kovtyukh, V. V., Gieren, W., \& Graczyk, D. 2011, AJ, 142, 51

Lutz, T. E., \& Kelker, D. H. 1973, PASP, 85, 573

Macri, L. M., Stanek, K. Z., Bersier, D., Greenhill, L. J., \& Reid, M. J. 2006, ApJ, 652, 1113

Maddrill, J. D. 1906, PASP, 18, 252

Madore, B. F. 1975, ApJS, 29, 219

Majaess, D. J., Turner, D. G., Lane, D. J., \& Krajci, T. 2011, JAVSO, 39, 219

Majaess, D., Turner, D., \& Gieren, W. 2012a, ApJ, 747, 145

Majaess, D., Turner, D., Gieren, W., Balam, D., \& Lane, D. 2012b, ApJ, 748, L9

Majaess, D. J., Turner, D. G., Gallo, L. C., et al. 2012c, ApJ, 753, 144

Martin, W. L., \& Warren, P. R. 1979, South Afr. Astr. Obs. Circ, No. 4, 98

Matsunaga, N., Feast, M. W., \& Soszyński, I. 2011, MNRAS, 413, 223

McGonegal, R., McAlary, C. W., McLaren, R. A., \& Madore, B. F. 1983, ApJ, 269,641

Mérand, A., Kervella, P., Coudé du Foresto, V., et al. 2005, A\&A, 438, L9

Mermilliod, J.-C., Mayor, M., \& Burki, G. 1987, A\&AS, 70, 389

Metzger, M. R., Caldwell, J. A. R., \& Schechter, P. L. 1992, AJ, 103, 529

Metzger, M. R., Caldwell, J. A. R., McCarthy, J. K., \& Schechter, P. L. 1993, ApJS, 76, 803

Moffett, T. J., \& Barnes, T. G. 1984, ApJS, 55, 389

Moffett, T. J., Gieren, W. P., Barnes, T. G., \& Gomez, M. 1998, ApJS, 117, 135

Monson, A. J., \& Pierce, M. J. 2011, ApJS, 193, 12

Molinaro, R., Ripepi, V., Marconi, M., et al. 2011, MNRAS, 413, 942

Molinaro, R., Ripepi, V., Marconi, M., et al. 2012, ApJ, 748, 69

Moore, J. H. 1909, Lick Obs. Bull., 5, 111

Mucciarelli, A., Cristallo, S., Brocato, E., et al. 2011, MNRAS 413, 837

Nardetto, N., Mourard, D., Kervella, P., et al. 2006, A\&A, 453, 309

Nardetto, N., Mourard, D., Mathias, Ph., Fokin, A., \& Gillet, D. 2007, A\&A, 471,661
Nardetto, N., Gieren, W., Kervella, P., et al. 2009, A\&A, 502, 951

Nardetto, N., Fokin, A., Fouqué, P., et al. 2011, A\&A, 534, L16

Ngeow, C., Kanbur, S. M., \& Nanthakumar, A. 2008, A\&A, 477, 621

Ngeow, C.-C., Kanbur, S. M., Neilson, H. R., Nanthakumar, A., \& Buonaccorsi, J. 2009, ApJ, 693, 691

Ngeow, C.-C., Neilson, H. R., Nardetto, N., \& Marengo, M. 2012, A\&A, accepted [arXiv: 1206. 1895]

Nikolaev, S., Drake, A. J., Keller, S. C., et al. 2004, ApJ, 601, 260

Niva, G. D., \& Schmidt, E. G. 1979, ApJ, 234, 245

Nordgren, T. E., Lane, B. F., Hindsley, R. B., \& Kervella, P. 2002, AJ, 123, 3380

Pejcha, O., \& Kochanek, S. 2012, ApJ, 748, 107

Pedicelli, S., Lemasle, B., Groenewegen, M. A. T., et al. 2010, A\&A, 518, A18

Pel, J. W. 1976, A\&AS, 24, 413

Pel, J. W. 1985, in Cepheids: Theory and Observations, ed. B. Madore, IAU Colloq. 82 (Cambridge: Cambridge Univ. Press), 1

Petroni, S., Bono, G., Marconi, M., \& Stellingwerf, R. F. 2003, ApJ, J599, 522

Petterson, O. K. L., Cottrell, P. L., \& Albrow, M. D. 2004, MNRAS, 350, 95

Petterson, O. K. L., Cottrell, P. L., Albrow, M. D., \& Fokin, A. 2005, MNRAS, 362,1167

Persson, S. E., Madore, B. F., Krzemiński, W., et al. 2004, AJ, 128, 2239

Pont, F., Burki, G., \& Mayor, M. 1994, A\&AS, 105, 165

Pont, F., Queloz, P., Bratschl, P., \& Mayor, M. 1997, A\&A, 318, 416

Press, W. H., Teukolsky, S. A., Vetterling, W. T., \& Flannery, B. P. 1992, in Numerical Recipes in Fortran (Cambridge University Press), 77

Rastorgouev, A. S., Gorynya, N. A., \& Samus, N. N. 1997, in Binary Stellar Systems, ed. A. G. Massevich (Moscow: Kozmosinform), 123

Riess, A. G., Macri, L., \& Li, W. 2009, ApJS, 183, 109

Ripepi, V., Moretti, M. I., Marconi, M., et al. 2012, MNRAS, 424, 1807

Romaniello, M., Primas, F., Mottini, M., et al. 2008, A\&A, 488, 731

Romeo, G., Fusi Pecci, F., \& Tosi, M. 1989, MNRAS, 240, 459

Sanford, R. F. 1927, ApJ, 66, 170

Sanford, R. F. 1935, ApJ, 81, 140

Sanford, R. F. 1951, ApJ, 114, 331

Sanford, R. F. 1956, ApJ, 123, 201

Sasselov, D. D., Beauliau, J. P., Renault, C., et al. 1997, A\&A, 324, 471

Scowcroft, V., Bersier, D., Mould, J. R., \& Wood, P. R. 2009, MNRAS, 396, 1287

Schechter, P. L., Avruch, I. M., Caldwell, J. A. R., \& Keane, M. J. 1992, AJ, 104 1930

Schmidt, E. G., \& Seth, A. 1996, AJ, 112, 2769

Schwarz, G. 1978, Ann. Stat., 6, 461

Shappee, B. J., \& Stanek, K. Z. 2011, ApJ, 733, 124

Shobbrook, R. R. 1992, MNRAS, 255, 486

Slipher, V. M. 1904, ApJ, 20, 146

Soszyński, I., Gieren, W., Pietrzyński, G., et al. 2005, PASP, 117, 823

Soszyński, I., Poleski, R., Udalski, A., et al. 2008, Acta Astron., 58, 163

Soszyński, I., Poleski, R., Udalski, A., et al. 2010, Acta Astron., 60, 17

Stibbs, D. W. N. 1955, MNRAS, 115, 363

Storm, J., Carney, B. W., Gieren, W. P., et al. 2004a, A\&A, 415, 521

Storm, J., Carney, B. W., Gieren, W. P., et al. 2004b, A\&A, 415, 531

Storm, J., Gieren, W. P., Fouqué, Barnes, T. G., \& Gómez, M. 2005, A\&A, 440, 487

Storm, J., Gieren, W., Fouqué, P., et al. 2011a, A\&A, 534, A94

Storm, J., Gieren, W., Fouqué, P., et al. 2011b, A\&A, 534, A95

Struve, O. 1945, ApJ, 102, 232

Sugars, B. J. A., \& Evans, N. R. 1996, AJ, 112, 1670

Szabados, L. 1977, Mitt. Sternw. Ung. Akad. Wiss., Budapest, No. 70

Szabados, L. 1980, Commun. Konkoly Obs. Hung. Acad. Sci., Budapest, No. 76

Szabados, L. 1981, Commun. Konkoly Obs. Hung. Acad. Sci., Budapest, No. 77

Szabados, L. 1989, CoKon, 94, 1

Szabados, L. 1990, MNRAS, 242, 285

Szabados, L. 1991, Commun. Konkoly Obs. Hung. Acad. Sci., Budapest, No. 96 Szabados, L. 1996, A\&A, 311, 189

Szabados, L. 2003, Inf. Bull. Var. Stars, No. 5394

Szabados, L., \& Pont, F. 1998, A\&AS, 133, 51

Tammann, G. A., Sandage, A., \& Reindl, B. 2003, A\&A, 404, 423

Taylor, M. M., \& Booth, A. J. 1998, MNRAS, 298, 594

Taylor, M. M., Albrow, M. D., Booth, A. J., \& Cottrell, P. L. 1997, MNRAS, 292,662

ten Bruggencate, P. 1930, Harvard Circ., no. 351, 1

Testa, V., Marconi, M., Musella, I., et al. 2007, A\&A, 462, 599

Turner, D. G. 1976, AJ, 81, 1125

Turner, D. G. 1977, AJ, 82, 163

Turner, D. G. 1979a, PASP, 91, 642

Turner, D. G. 1979b, A\&A, 76, 350

Turner, D. G. 1986, AJ, 92, 111

Turner, D. G. 1992, AJ, 104, 1865

Turner, D. G. 1998, AJ, 116, 274 
Turner, D. G. 2010, Astrophys. Space Sci., 326, 219

Turner, D. G. 2011, Rev. Mex. Astron. Astrofis., 47, 127

Turner, D. G., \& Burke, J. F. 2002, AJ, 124, 2931

Turner, D. G., Forbes, D., \& Pedreros, M. 1992, AJ, 104, 1132

Turner, D. G., Van Den Bergh, S., Younger, F. P., Danks, T. A., \& Forbes, D. 1993, ApJS, 85, 119

Turner, D. G., Pedreros, M., \& Walker, A. R. 1998, AJ, 115, 1958

Turner, D. G., Bryukhanov, I. S., Balyuk, I. I., et al. 2007, PASP, 119, 1247

Turner, D. G., Majaess, D. J., Lane, D. J., et al. 2010, OAP, 23, 119

Udalski, A., Soszyński, I., Szymański, M., et al. 1999, AcA, 49, 223

van Belle, G. T., Lane, B. F., \& Thompson, R. R. 1999, AJ, 117, 521

van der Marel, R. P., \& Cioni, M.-R. 2001, AJ, 122, 1807

van Genderen, A. M. 1983, A\&AS, 52, 423

van Leeuwen, F. 2009, A\&A, 497, 209

van Leeuwen, F., Feast, M. W., Whitelock, P. A., \& Laney, C. D. 2007, MNRAS, 379,723

Vinkó, J. 1993, MNRAS, 260, 273

Walker, A. R. 1985a, MNRAS, 213, 889

Walker, A. R. 1985b, MNRAS, 214, 45
Walker, A. R. 1987a, MNRAS, 225, 627

Walker, A. R. 1987b, SAAOC, 11, 131

Walker, A. R. 1987 c, MNRAS, 229, 31

Walker, A. R. 2012, 2012, Ap\&SS, 341, 43

Wallerstein, G. 1972, PASP, 84, 656

Walraven, J. H., Tinbergen, J., \& Walraven, T. 1964, BAN, 17, 520

Welch, D. L. 1985, Ph.D. Thesis, University of Toronto

Welch, D. L., \& Evans, N. E. 1989, AJ, 97, 1153

Welch, D. L., Wieland, F., McAlary, C. W., et al. 1984, ApJS, 54, 547

Welch, D. L., Evans, N. R., Lyons, R. W., et al. 1987a, PASP, 99, 610

Welch, D. L., McLaren, R. A., Madore, B. F., \& McAlary, C. W. 1987b, ApJ, 321,162

Welch, D. L., Mateo, M., Cote, P., Fischer, P., \& Madore, B. F. 1991, AJ, 101, 490

Wilson, T. D., Carter, M. W., Barnes, T. G., Van Citters, G. W., \& Moffett, T. J. 1989, ApJS, 69, 951

Wisniewski, W. Z., \& Johnson, H. R. 1968, CoLPL, 7, 57

Yong, D., Carney, B. W., Teixera de Almeida, M.-L., \& Pohl, B. L. 2006, AJ, 131,2256 
M. A. T. Groenewegen: Baade-Wesselink distances and the effect of metallicity in Cepheids

Table 1. Sources of $V$-, $K$-band and RV data for the Galactic Cepheids.

\begin{tabular}{|c|c|c|c|c|}
\hline Name & $V$ & $K$ & RV & Data not considered $^{a}$ \\
\hline AK Cep & 3 & 111 & 33 & \\
\hline AN Aur & 3 & 111 & 33,39 &,,-- 39 \\
\hline AQ Pup & $1,2,3,4,5,84,99$ & $6,7,8$ & $1,4,39,44,113$ & $3,-,-\mathrm{JD}<44100, \mathrm{JD}>48500, \dot{P}$ \\
\hline AV Sgr & $3,16,112$ & 112 & $16,26,41,112$ & $\mathrm{JD}<45000$ \\
\hline AW Per & $2,3,13,27,30,116$ & 111 & $9,28,33,39,117,118,119$ &,,$-- 9 \mathrm{JD}<40000$ \\
\hline BB Sgr & $2,3,16,23,63$ & 6,24 & $20,33,39,44,47,113$ &,,$-- 44 \mathrm{JD}<44400$ \\
\hline BE Mon & 3,125 & 111 & 4 & \\
\hline beta Dor & $3,11,16,35$ & 6,18 & $11,19,20,21,22,47$ &,,$-- 20 \mathrm{JD}<40000$ \\
\hline BF Oph & $2,3,16,23,63$ & $6,18,24$ & $10,20,39,44,76$ & $\mathrm{JD}<36000$ \\
\hline BG Lac & $2,3,12,13$ & 12,111 & $14,15,39,44$ & $\mathrm{JD}<33000$ \\
\hline BM Per & 3,120 & 111 & 33,121 & \\
\hline BN Pup & $1,3,5,16$ & 6,7 & 1,17 & $\mathrm{JD}<33000$ \\
\hline BZ Cyg & $3,64.120$ & 111 & 33 & \\
\hline CD Cyg & $2,3,64$ & 24,111 & 33,44 & \\
\hline CF Cas & 2,3 & 111 & $4,17,33,105$ & \\
\hline CK Sct & 3,16 & 111 & $17,26,105$ & \\
\hline CP Cep & 3 & 111 & $17,33,105$ & \\
\hline CR Cep & 2,3 & 111 & $4,44,105$ &,,-- 44 \\
\hline CR Ser & 3 & 111 & 33 & \\
\hline CS Vel & 3,120 & $6,7,24$ & 4,26 & $-, 24,-$ \\
\hline CV Mon & $2,3,13,16,23$ & $6,24,111$ & $25,26,44$ & \\
\hline DD Cas & $2,3,13$ & 111 & $4,33,44$ & \\
\hline delta Cep & $2,3,11,12,13,27$ & 12,98 & $4,9,14,25,28,29,37,39$ &,,$-- 9 \mathrm{JD}<43500$ \\
\hline DL Cas & $2,3,11,13$ & 111 & $4,33,44,105,122$ & $11,-,-\mathrm{JD}<40000$ \\
\hline DT Cyg & $2,3,4,27,30,31$ & 24,32 & $4,9,28,29,33,79$ & $-, 32,9+28 \mathrm{JD}<33400$ \\
\hline eta Aql & $2,3,12,13,16,23,27,35$ & 12,24 & $9,14,20,25,28,29,36,37$ &,,-- 9 \\
\hline FF Aql & $2,3,11,16,23,27,30,31$ & $18,24,32$ & $9,20,28,29,33,38$ & $3,32,-\mathrm{JD}<44400$ \\
\hline FM Aql & $2,3,12,13,16,23$ & $12,24,111$ & $14,33,39,44$ & $\mathrm{JD}<30000$ \\
\hline
\end{tabular}

Notes. ${ }^{(a)}$ The number indicates the dataset not used in $V, K, \mathrm{RV}$, respectively.

References. 1 = Coulson \& Caldwell (1985a); 2 = Moffett \& Barnes (1984); 3 = Berdnikov et al. (2000), a datafile named "cepheids-16-03-2006" was retrieved from the ftp address listed in that paper; $4=$ Bersier et al. (1994); 5 = Madore (1975); $6=$ Laney \& Stobie (1992); $7=$ Schechter et al. (1992); 8 = Welch (1985); 9 = Barnes et al. (1987), points with uncertainty flag “:” were removed; $10=$ Stibbs (1955); 11 = Bersier (2002), data points with weight 0 and 1 in the Geneva photometry were removed; $12=$ Barnes et al. (1997); $13=$ Szabados $(1980) ; 14=$ Barnes et al (2005a,b); 15 = Imbert (1999); 16 = Pel (1976); 17 = Pont et al. (1994); 18 = Lloyd Evans (1980a); $19=$ Nardetto et al. (2006); 20 = Lloyd Evans (1980b); 21 = Taylor \& Booth (1998); 22 = Petterson et al. (2005); $23=$ Caldwell et al. (2001); $24=$ Welch et al. (1984); $25=$ Storm et al. (2004a,b); 26 = Metzger et al. (1992); 27 = Kiss (1998); 28 = Wilson et al. (1989); 29 = Kiss (2000); 30 = Szabados (1991); 31 = Szabados (1977); 32 = Wisniewski \& Johnson (1968); 33 = Gorynya et al. (1998, VizieR On-line Data Catalog: III/229); $34=$ Sanford (1935); $35=$ Shobbrook (1992); 36 = Jacobsen \& Wallerstein (1981); 37 = Evans (1976); 38 = Evans et al. (1990); 39 = Joy (1937); $40=$ Walraven et al. (1964); 41 = Grayzeck (1978); 42 = Groenewegen (2008); 43 = Taylor et al. (1997); 44 = Barnes et al. (1988), points with uncertainty flag "“:” were removed; 45 = Turner et al. (2007); 46 = Duncan (1908); 47 = Lloyd Evans (1968); 48 = Petterson et al. (2004); $49=$ Campbell \& Moore (1928); 50 = Böhm-Vitense et al. (1990); 51 = Mermilliod et al. (1987); $52=$ Feast (1967); 53 = Breger (1970); $54=$ Evans et al. (1993); $55=$ Herbig \& Moore (1952); 56 = Breitfellner \& Gillet (1993); 57 = Adams \& Shapley (1918); $58=$ Abt (1959); $59=$ Niva \& Schmidt (1979); $60=$ Gieren (1976); 61 = Häupl (1988); 62 = Beavers \& Eitter (1986); 63 = Gieren (1981b); 64 = Szabados (1981); 65 = Sanford (1956); $66=$ Evans et al. (1999); 67 = Frost (1906); 68 = Sanford (1927); $69=$ Wallerstein (1972); $70=$ Coulson (1983); 71 = Evans \& Lyons (1994); $72=$ Gieren (1989); 73 = Harper (1934); $74=$ Coulson et al. (1985); $75=$ Gieren (1985); $76=$ Gieren (1981a); 77 = Jacobsen (1970); $78=$ Breger (1967); $79=$ Sanford (1951); $80=$ Imbert (1996); 81 = Coulson \& Caldwell (1985b); 82 = Eggen (1983); 83 = Evans \& Sugars (1997); 84 = HIPPARCos Epoch Photometry; 85 = Babel et al. (1989); 86 = Kimeswenger et al. (2004); 87 = Albrow \& Cottrell (1996); $88=$ Jacobsen et al. (1984); 89 = Jacobsen (1974); 90 = Henden (1980); 91 = Evans \& Lyons (1992); 92 = Abt \& Levy (1978); 93 = Duncan (1922); 94 = ten Bruggencate (1930); 95 = Evans \& Welch (1993); 96 = Gieren (989); 97 = Sugars \& Evans (1996); 98 = Fernley et al. (1989); $99=$ Dean (1977); 100 = Dean (1981); 101 = Duncan (1932); 102 = Slipher (1904); 103 = Moore (1909); 104 = Feast et al. (2008); $105=$ Metzger et al. (1993); $106=$ Evans (1988); 107 = Maddrill (1906); 108 = Abt (1973); $109=$ Imbert (1985); $110=$ Joy (1952); $111=$ Monson \& Pierce (2011); $112=$ Pedicelli et al. (2010); $113=$ Storm et al. (2011a); $114=$ This paper (Table 3); $115=$ McGonegal et al. (1983); $116=$ Vinkó (1993); $117=$ Evans $(1983)$; $118=$ Evans et al. (2000); $119=$ Welch \& Evans (1989); $120=$ Harris (1980); 121 = Pont et al. (1997); $122=$ Gieren et al. (1994); $123=$ Struve (1945); 124 = Welch et al. (1987a,b); 125 = Schmidt \& Seth (1996); 126 = Szabados \& Pont (1998); 127 = Kienzle et al. (1999). 
Table 1. continued.

\begin{tabular}{|c|c|c|c|c|}
\hline Name & $V$ & $K$ & RV & Data not considered $^{a}$ \\
\hline FM Cas & $2,3,13$ & 111 & $4,33,44$ &,,-- 44 \\
\hline FN Aql & $2,3,12,13,16,23$ & $12,24,111$ & $14,33,39$ &,,-- 39 \\
\hline GH Lup & $1,3,16,23$ & 6 & 1 & $\mathrm{JD}<43000$ \\
\hline GY Sge & 3 & 6,111 & 33,105 & $\mathrm{JD}<45500, \mathrm{JD}>48200, \dot{P}$ \\
\hline KN Cen & $1,3,5,11,16,40,84$ & 6 & $1,17,41$ & $-,-, 41 \mathrm{JD}<40000, \mathrm{JD}>47000$ \\
\hline KQ Sco & $1,3,11$ & 6 & $1,42,114$ & \\
\hline $1 \mathrm{Car}$ & $3,5,11,16,35$ & 6 & $11,19,20,23,43,47$ & $5,-, 23 \mathrm{JD}<46500$ \\
\hline LS Pup & $1,3,11$ & 6 & $1,113,114$ &,,-- 1 \\
\hline MW Cyg & $2,3,13$ & 111 & $33,44,80$ &,,-- 44 \\
\hline QZ Nor & $1,3,11$ & 6 & $26,81,114,127$ &,,-- 81 \\
\hline RR Lac & $2,3,13,120$ & 111 & $4,33,44$ &,,-- 44 \\
\hline RS Cas & 3 & 111 & 33,39 &,,-- 39 \\
\hline RS Ori & $2,3,13,16,30$ & 111 & 33 & \\
\hline RS Pup & $2,3,5,11,16,84$ & $6,8,24$ & $11,19,25,44$ & $-,-, 44 \mathrm{JD}<42000, \mathrm{JD}>50000, \dot{P}$ \\
\hline RT Aur & $2,12,27,31,45$ & $12,32,111$ & $9,28,29,33,37,46$ & $-, 32,9 \mathrm{JD}<40000$ \\
\hline RU Sct & $2,3,16$ & 6,111 & $26,33,39,105$ &,,$-- 39 \mathrm{JD}<25000$ \\
\hline RW Cam & $2,3,30,64$ & 111 & 44 & \\
\hline RW Cas & $2,3,64$ & 111 & 33,44 & \\
\hline RX Aur & $2,3,64$ & 111 & $28,33,44$ & \\
\hline RX Cam & $2,3,13$ & 111 & $33,44,80$ & $\mathrm{JD}<40000$ \\
\hline RY Cas & 3,30 & 111 & 33 & \\
\hline $\mathrm{RY}$ CMa & $2,3,16$ & 111 & 33,44 & \\
\hline RY Sco & $1,2,3,5,11,16$ & 6 & $1,20,44$ & \\
\hline RY Vel & $1,3,5,11,16$ & 6 & $1,11,17$ & $\mathrm{JD}<44000, \mathrm{JD}>50000, \dot{P}$ \\
\hline $\mathrm{RZ} \mathrm{CMa}$ & $2,3,16$ & 111 & 17,44 & \\
\hline RZ Gem & $2,3,13$ & 111 & 33,44 & \\
\hline RZ Vel & $1,3,5,11,16$ & 6 & $1,11,20,47$ & \\
\hline S Mus & $3,11,23,40$ & 6,24 & $10,11,20,22,41,47,48,49,50$ &,,$-- 41 \mathrm{JD}<30000$ \\
\hline S Nor & $3,4,23,40$ & 6,24 & $4,10,20,41,47,49,51,52,53$ &,,$-- 41 \mathrm{JD}<30000$ \\
\hline S Sge & $2,3,11,12,13,23,27,30,40$ & $12,24,32$ & $9,14,28,29,33,37,54,55,56$ & $-, 32,9+28+54$ \\
\hline SS Sct & $2,3,16,23,63$ & 24,111 & $39,42,44,63,114$ & $\mathrm{JD}<30000$ \\
\hline ST Tau & $2,3,12,31,90$ & 111 & $4,33,44$ & $90,-,-$ \\
\hline SU Cas & $2,3,9,27,31$ & 12,32 & $4,9,25,28,29,33,57,58,59,60,61,62$ & $-, 32,9+28+59+61+62 \mathrm{JD}<43000$ \\
\hline SU Cyg & $2,3,27,30,31$ & 24,111 & $9,28,33,106,107,108,109$ &,,$-- 9 \mathrm{JD}<35000$ \\
\hline SV Mon & $1,2,3,64,90$ & 111 & $1,33,44$ & \\
\hline SV Per & $2,3,64,90$ & 111 & $33,44,121$ &,,-- 44 \\
\hline S Vul & 3,30 & 6,111 & 33,110 & $\mathrm{JD}<45400, \mathrm{JD}>49200, \dot{P}$ \\
\hline SV Vul & $2,3,4,9,27,64$ & $6,12,24,111$ & $4,9,14,15,25,28,29,33,39,65$ & $\mathrm{JD}<45500, \mathrm{JD}>48600, \dot{P}$ \\
\hline SW Cas & $2,3,13$ & 111 & 33 & \\
\hline SW Vel & $1,3,5,11,16,84$ & 6 & 1,11 & $\mathrm{JD}>48000, \dot{P}$ \\
\hline SX Vel & $3,23,40$ & 6 & 10 & \\
\hline SY Cas & 3,31 & 111 & 4,33 & \\
\hline SZ Aql & $2,3,11,12,16,23,64$ & $6,12,24,111$ & $11,14,23$ & \\
\hline SZ Cyg & $2,3,64$ & 111 & $39,44,123$ & $\mathrm{JD}<30000$ \\
\hline SZ Tau & $2,3,4,12,27,31$ & $6,12,32$ & $4,9,28,29,33$ & $-, 32,9 \mathrm{JD}<43000, \mathrm{JD}>48000, \dot{P}$ \\
\hline T Mon & $1,2,3,11,16,35,64$ & $6,24,32,111$ & $4,9,25,29,33,37,48,65,66,67,68,69,70,71,72,73$ & $-, 32,70 \mathrm{JD}<44500$ \\
\hline TT Aql & $2,3,11,12,16,27,64,74$ & $12,24,111$ & $9,11,14,15,22,25,28,33,37,39,74$ &,,$-- 74 \mathrm{JD}<47000$ \\
\hline T Vel & $3,16,75$ & 6,18 & $10,20,75$ & $-, 18,-$ \\
\hline T Vul & $2,3,4,12,27,31$ & $12,24,32,98$ & $4,9,14,28,29,37$ & $-, 32,9 \mathrm{JD}<43600$ \\
\hline
\end{tabular}


M. A. T. Groenewegen: Baade-Wesselink distances and the effect of metallicity in Cepheids

Table 1. continued.

\begin{tabular}{|c|c|c|c|c|}
\hline Name & $V$ & $K$ & RV & Data not considered $^{a}$ \\
\hline TW Nor & $3,5,16,120$ & 6,24 & 4,26 & \\
\hline TY Sct & 3,16 & 111 & $26,39,105$ &,,-- 39 \\
\hline TZ Mon & $3,16,121$ & 111 & $17,39,121$ & $\mathrm{JD}<40000$ \\
\hline U Aql & $2,3,16,27$ & 111 & $9,11,20,28,124$ &,,$-- 44 \mathrm{JD}<42000$ \\
\hline U Car & $1,3,5,11,16,35$ & 6,24 & $1,11,20$ & \\
\hline U Nor & $1,5,11,16$ & 6 & $1,11,17,41$ & \\
\hline U Sgr & $2,3,4,16,23,35,63$ & $6,24,32,111,115$ & $4,9,10,11,20,25,28,33,39,47,51,76,77,78$ & $-, 32,9 \mathrm{JD}<37000$ \\
\hline UU Mus & $1,5,11,40,84$ & 6 & 1,11 & \\
\hline U Vul & $2,3,12,13,23,27$ & 12,111 & $4,14,33,37,44,79,80$ &,,-- 44 \\
\hline UZ Sct & $3,5,16,112$ & 111,112 & $17,26,39,105,112$ & $\mathrm{JD}<47500$ \\
\hline V1162 Aql & 3 & 111 & 33,114 & \\
\hline V340 Ara & $3,5,23$ & 112 & $17,23,112$ & $\mathrm{JD}<50000$ \\
\hline V340 Nor & $3,4,82$ & 6 & $4,26,114$ & \\
\hline V350 Sgr & $2,3,23,63,99$ & 18,24 & $20,22,33,39,44,76,83,114$ & $-, 18,76 \mathrm{JD}<42000$ \\
\hline V386 Cyg & $2,3,13$ & 111 & $33,44,105$ & \\
\hline V402 Cyg & $2,3,31,90$ & 111 & 33,105 & $90,-,-$ \\
\hline V459 Cyg & 2,3 & 111 & $17,33,105$ & \\
\hline V495 Cyg & 3,125 & 111 & 33,105 & \\
\hline V496 Aql & $2,3,16,63$ & 24 & $9,10,20,33,76,113,114$ &,,$-- 9 \mathrm{JD}<40500$ \\
\hline V538 Cyg & 3 & 111 & 33 & \\
\hline V600 Aql & $2,3,13,16$ & 111 & $17,33,105$ & \\
\hline V916 Aql & 3 & 111 & 33 & \\
\hline V Car & $3,16,23$ & 6 & $10,20,47$ & \\
\hline V Cen & $3,11,16,23,63$ & 6,24 & $10,11,20,47,76$ & $-, 24,-\mathrm{JD}<40000$ \\
\hline VW Cen & $1,3,16,84$ & 6 & $1,41,114$ &,,-- 41 \\
\hline VX Cyg & $3,64,120$ & 111 & 33,39 & $\mathrm{JD}<30000$ \\
\hline VX Per & $2,3,30,84,120$ & 111 & $33,44,105$ & $30,-,-$ \\
\hline VY Car & $1,3,5,11,16,84,99,100$ & 6,24 & $1,11,20,41$ & $84,-,-$ JD $<42000$, JD $>50490, \dot{P}$ \\
\hline VY Cyg & 2,3 & 111 & $39,44,123$ & $\mathrm{JD}<40000$ \\
\hline VY Sgr & $3,16,112$ & 112 & $17,26,39,112$ & $\mathrm{JD}<47000$ \\
\hline VZ Cyg & $2,3,12,31$ & $12,24,111$ & $4,14,33,39,44,113$ & $\mathrm{JD}<40000$ \\
\hline VZ Pup & $1,3,5,11,16$ & $6,7,8$ & $1,11,113$ & \\
\hline W Gem & $2,3,13,16,27$ & 111 & $9,28,33$ & \\
\hline W Sgr & $2,3,23,35,40,85$ & $24,32,86$ & $4,9,10,20,22,28,47,48,85,87,88,89$ &,,$-- 9+28 \mathrm{JD}<39000$ \\
\hline WZ Car & $1,3,5,11,16$ & 6 & 1,11 & \\
\hline WZ Sgr & $1,2,3,5,11,40$ & 6,111 & $1,11,33,39$ & $\mathrm{JD}<40000$ \\
\hline X Cyg & $2,3,4,27,64$ & $12,24,32,98$ & $4,14,25,29,33,37$ & $-, 32,-$ \\
\hline X Lac & $2,3,12,13$ & 12,111 & $4,14,33,44$ & $-, 12,44$ \\
\hline X Pup & $2,3,5,11,16,23$ & 6 & $11,23,44,113$ & $5,-,-$ \\
\hline X Sgr & $2,3,11,16,23,35,99$ & $24,32,104$ & $9,10,11,20,28,47,52,101,102,103,113$ & $-, 32,9 \mathrm{JD}<48800$ \\
\hline $\mathrm{X}$ Vul & $2,3,13$ & 111 & $4,33,44$ &,,-- 44 \\
\hline XX Cen & $3,11,40,74$ & 6,24 & $10,11,20,41,52,74$ & $-, 24,41$ \\
\hline XX Sgr & $2,3,23$ & 24 & 113,114 & JD $<44000$ \\
\hline Y Lac & $2,3,12,31,90$ & 12,111 & $14,15,33,39,44$ & $-, 111,-\mathrm{JD}<40000$ \\
\hline Y Oph & $1,2,3,16$ & 6,24 & $1,9,19,20,28,33,34,91,92$ &,,$-- 9 \mathrm{JD}<40000$ \\
\hline Y Sct & $2,3,16$ & 111 & 11,44 & \\
\hline Y Sgr & $2,3,11,16,23,35$ & 24,32 & $9,11,19,20,28,46,49,93,94,113,114$ & $-, 32,9 \mathrm{JD}<28500$ \\
\hline YZ Aur & 3,64 & 111 & $3,39,126$ & $\mathrm{JD}<45000$ \\
\hline YZ Sgr & $2,3,23,40$ & 24,111 & $20,44,113$ & JD $<44900$ \\
\hline zeta Gem & $2,3,4,27,35,64$ & 32,104 & $4,9,19,28,29,33,37,113$ & $-, 32,9 \mathrm{JD}<23500$ \\
\hline Z Lac & $2,3,12,64$ & 12,111 & $14,33,39,44,80,95,96,97$ & $\mathrm{JD}<40500$ \\
\hline
\end{tabular}


Table 2. Sources of $V$-, $K$-band and RV data for the MC Cepheids.

\begin{tabular}{|c|c|c|c|c|}
\hline Name* & $V$ & $K$ & RV & Data not considered $^{a}$ \\
\hline HV $822^{*}$ & $1001,1002,1003$ & 1001 & 1001 & $1002,-,--$ \\
\hline HV $837^{*}$ & $1002,1003,1004,1005,1006,1007$ & 1008,1009 & 1010 & \\
\hline HV 873 & 1002,1004 & 1009,1011 & 1012 & $-, 1009,-$ \\
\hline HV 876 & 1004 & 1011 & 1012 & \\
\hline HV 877 & $1002,1003,1004,1005,1006$ & $1008,1009,1011$ & 1012 & \\
\hline HV 878 & 1002,1004 & 1009,1011 & 1012 & \\
\hline HV 879 & 1004, 1006, 1007 & $1008,1009,1011$ & 1010 & $-, 1009,-$ \\
\hline HV 881 & $1002,1004,1005$ & 1009,1011 & 1012 & \\
\hline HV 899 & $1002,1004,1006,1007$ & 1009,1011 & 1010 & $1006,1009,-$ \\
\hline HV 900 & 1002, 1003, 1004, 1005, 1007 & 1009,1011 & 1010,1012 &,,--- JD $<44600 \mathrm{JD}<51000$ \\
\hline HV 909 & $1002,1003,1006,1007$ & 1009,1011 & 1010 & $-, 1009,-$ \\
\hline HV 914 & 1004 & 1009,1011 & 1012 & $-, 1009,-$ \\
\hline HV 1005 & 1004,1006 & 1011 & 1012 & \\
\hline HV 1006 & 1004 & 1011 & 1012 & \\
\hline HV 1023 & 1004,1006 & 1011,1008 & 1012 & \\
\hline HV $1328^{*}$ & 1001, 1003, 1004 & 1001,1009 & 1001 &,,$---\mathrm{JD}<47000$ \\
\hline HV 1333* & 1001,1004 & 1001 & 1001 & \\
\hline HV $1335^{*}$ & 1001,1004 & 1001 & 1001 & \\
\hline HV $1345^{*}$ & 1001,1004 & 1001 & 1001 & \\
\hline HV 2257 & $1002,1004,1005,1007$ & 1009,1011 & 1010 & $-, 1009,-\mathrm{JD}<44500, \mathrm{JD}>50500, \dot{P}$ \\
\hline HV 2282 & 1004 & 1011 & 1012 & \\
\hline HV 2338 & $1002,1005,1006,1007$ & 1009,1011 & 1010 & $-, 1009,-$ \\
\hline HV 2369 & $1002,1003,1004,1005,1006,1013$ & 1009,1011 & 1012 & $\mathrm{JD}<44300, \dot{P}$ \\
\hline HV 2405 & 1004 & 1009,1011 & 1012 & \\
\hline HV 2527 & 1004,1006 & 1009,1011 & 1012 & \\
\hline HV 2538 & 1004 & 1011 & 1012 & \\
\hline HV 2549 & $1003,1004,1006$ & 1009,1011 & 1012 & \\
\hline HV 2827 & 1003, 1006, 1007 & $1008,1009,1011$ & 1010 & $-, 1008,-$ \\
\hline HV 5655 & 1004 & 1011 & 1012 & \\
\hline HV 6093 & 1004 & 1011 & 1012 & \\
\hline HV 12197 & $1014,1015,1016,1017$ & 1018 & $1015,1018,1022$ & $\mathrm{JD}<37000$ \\
\hline HV 12198 & $1014,1015,1016,1017$ & 1018 & 1001, 1016, 1018 & $\mathrm{JD}<37000$ \\
\hline HV 12199 & $1014,1015,1016,1017$ & 1018 & $1015,1018,1022$ & $\mathrm{JD}<37000$ \\
\hline HV 12202 & 1014, 1015, 1016, 1017 & 1018,1019 & 1015,1018 & $-, 1019,-$ JD $<37000$ \\
\hline HV 12203 & $1014,1015,1016,1017$ & 1018 & 1015,1018 & $\mathrm{JD}<37000$ \\
\hline HV 12204 & $1014,1015,1016$ & 1018 & 1015,1018 & $\mathrm{JD}<37000$ \\
\hline HV 12452 & 1004 & 1011 & 1012 & \\
\hline HV 12505 & 1004 & 1011 & 1012 & \\
\hline HV 12717 & 1004 & 1011 & 1012 & \\
\hline HV 12815 & $1003,1006,1007,1020$ & $1008,1009,1011$ & 1020 & $-, 1009,-$ \\
\hline HV 12816 & $1006,1007,1020$ & $1008,1009,1011$ & 1020,1021 &,,-- 1020 \\
\hline U 1 & 1004 & 1011 & 1018 & \\
\hline
\end{tabular}

Notes. ${ }^{(a)}$ The number indicates the dataset not used in $V, K, \mathrm{RV}$, respectively. ${ }^{(*)}$ Objects marked by a $\star$ are located in the SMC.

References. 1001 = Storm et al. (2004a,b); 1002 = Madore (1975); $1003=$ Van Genderen (1983); $1004=$ OGLE-III data (Soszynski et al. 2008, 2010); 1005 = Eggen (1977); 1006 = Martin \& Warren (1979); 1007 = Moffett et al. (1998); 1008 = Laney \& Stobie (1986); 1009 = Welch et al. (1987a,b); $1010=$ Imbert (1989); $1011=$ Persson et al. (2004); $1012=$ Storm et al. (2011b); $1013=$ Freedman et al. $(1985) ; 1014=$ Gieren et al. (2000); $1015=$ Welch et al. (1991); $1016=$ Walker (1987a); $1017=$ Arp \& Thackeray (1967); $1018=$ Storm et al. (2005); 1019 = Testa et al. (2007); $1020=$ Caldwell et al. (1986); $1021=$ Gieren et al. (2005); $1022=$ Molinaro et al. (2012). 
M. A. T. Groenewegen: Baade-Wesselink distances and the effect of metallicity in Cepheids

Table 3. New radial velocity data.

\begin{tabular}{|c|c|c|c|c|c|c|c|}
\hline JD & $\mathrm{RV}\left(\mathrm{km} \mathrm{s}^{-1}\right)$ & JD & $\mathrm{RV}\left(\mathrm{km} \mathrm{s}^{-1}\right)$ & $\mathrm{JD}$ & $\mathrm{RV}\left(\mathrm{km} \mathrm{s}^{-1}\right)$ & JD & $\mathrm{RV}\left(\mathrm{km} \mathrm{s}^{-1}\right)$ \\
\hline \multicolumn{2}{|c|}{ KQ Sco } & \multicolumn{2}{|c|}{ V340 Nor } & \multicolumn{2}{|c|}{ VW Cen } & \multicolumn{2}{|c|}{ Y Sgr } \\
\hline 2454625.7165 & -43.06 & 2454626.5964 & -47.27 & 2454626.5170 & -63.59 & 2454302.5565 & -9.03 \\
\hline 2454626.8125 & -47.14 & 2454626.6375 & -47.17 & 2454626.5863 & -63.60 & 2454303.7440 & 2.45 \\
\hline 2454627.5483 & -46.52 & 2454627.5619 & -45.44 & 2454627.5270 & -60.25 & 2454304.6563 & 10.42 \\
\hline 2454628.6980 & -45.57 & 2454628.6236 & -40.48 & 2454627.6088 & -59.83 & 2454305.7003 & 14.94 \\
\hline 2454631.5682 & -32.87 & 2454631.5810 & -29.51 & 2454631.5496 & -33.87 & 2454306.7067 & -20.91 \\
\hline 2454632.7716 & -28.10 & 2454633.6032 & -40.26 & 2454633.5906 & -21.36 & 2454307.6489 & -15.31 \\
\hline 2454633.6530 & -25.24 & 2454635.5536 & -45.34 & X S & & 2454308.6302 & -6.21 \\
\hline 2454953.6445 & -12.65 & \multicolumn{2}{|c|}{ V350 Sgr } & 2454302.7712 & 18.62 & 2454309.5865 & 2.96 \\
\hline \multicolumn{2}{|c|}{ LS Pup } & 2454302.5747 & 12.56 & 2454304.7842 & -6.32 & 2454310.7130 & 14.48 \\
\hline 2454627.4580 & 102.98 & 2454303.7480 & 25.54 & 2454305.7605 & 0.02 & 2454311.7705 & -0.97 \\
\hline 2454631.4584 & 66.99 & 2454304.7665 & 6.63 & 2454306.6959 & 13.83 & 2454312.7778 & -20.24 \\
\hline 2454635.4577 & 69.31 & 2454305.7794 & -7.51 & 2454307.7367 & 31.34 & 2454331.6950 & -6.40 \\
\hline \multicolumn{2}{|c|}{ QZ Nor } & 2454306.8017 & 2.13 & 2454309.7736 & -2.81 & 2454332.5063 & 1.38 \\
\hline 2454303.5850 & -31.84 & 2454307.7016 & 12.21 & 2454310.7698 & 12.04 & 2454334.5199 & 14.87 \\
\hline 2454307.6148 & -30.76 & 2454308.6363 & 21.25 & 2454312.7944 & 17.84 & 2454339.5444 & 13.17 \\
\hline 2454311.6411 & -30.72 & 2454309.6259 & 24.82 & 2454332.6218 & 27.46 & 2454341.5468 & -21.03 \\
\hline 2454334.5106 & -32.10 & 2454310.7948 & -8.45 & 2454335.6077 & 6.87 & 2454625.8749 & -8.96 \\
\hline 2454341.5283 & -31.64 & 2454311.6175 & -1.54 & 2454341.6291 & 31.67 & 2454626.8939 & 1.02 \\
\hline 2454625.5461 & -30.94 & 2454312.8122 & 11.59 & 2454626.6591 & 29.84 & 2454627.6650 & 7.31 \\
\hline 2454633.6613 & -32.27 & 2454331.7205 & -6.69 & 2454627.8317 & 2.80 & 2454630.8372 & -16.37 \\
\hline \multicolumn{2}{|c|}{ SS Sct } & 2454332.7166 & 3.26 & 2454630.8799 & 29.94 & 2454631.8182 & -7.29 \\
\hline 2454625.8712 & 7.07 & 2454334.6189 & 23.99 & 2454633.6960 & 7.21 & 2454632.8076 & 2.19 \\
\hline 2454627.6691 & -13.88 & 2454335.5375 & 15.52 & 2454953.7207 & 23.38 & 2454633.6681 & 9.60 \\
\hline 2454630.8872 & -21.58 & 2454339.6487 & 20.88 & $\mathrm{XX}$ & & 2454634.8012 & 14.13 \\
\hline 2454631.8791 & -5.11 & 2454341.6415 & -9.50 & 2454302.5656 & 23.35 & 2454953.7064 & -19.59 \\
\hline 2454632.8718 & 7.04 & 2454625.8994 & -8.11 & 2454303.7571 & 35.71 & & \\
\hline 2454633.6781 & -8.44 & 2454626.6336 & -0.31 & 2454304.6605 & -4.33 & & \\
\hline 2454634.8687 & -16.28 & 2454627.7019 & 10.97 & 2454305.7946 & -2.23 & & \\
\hline \multicolumn{2}{|c|}{ V1162 Aql } & 2454628.6315 & 20.74 & 2454306.7194 & 4.97 & & \\
\hline 2454302.7986 & 2.26 & 2454630.8919 & -9.61 & 2454307.6692 & 12.70 & & \\
\hline 2454304.8177 & 19.95 & 2454632.8342 & 10.67 & 2454308.6166 & 18.08 & & \\
\hline 2454305.8033 & 30.86 & 2454633.6831 & 19.28 & 2454309.7481 & 36.45 & & \\
\hline 2454306.8129 & 9.31 & \multicolumn{2}{|c|}{ V496 Aql } & 2454310.7181 & 9.30 & & \\
\hline 2454307.7181 & -0.40 & 2454304.7716 & 10.86 & 2454311.7645 & -4.85 & & \\
\hline 2454308.6830 & 6.64 & 2454302.5863 & -0.34 & 2454312.7287 & 1.62 & & \\
\hline 2454309.5984 & 15.19 & 2454302.5914 & -0.32 & 2454331.6906 & -0.65 & & \\
\hline 2454310.8143 & 26.18 & 2454303.7521 & 4.29 & 2454332.5108 & 5.84 & & \\
\hline 2454311.7788 & 25.46 & 2454305.7241 & 14.40 & 2454334.5262 & 20.30 & & \\
\hline 2454312.8241 & -0.59 & 2454306.7725 & 1.55 & 2454339.5496 & 10.37 & & \\
\hline 2454331.7254 & 19.79 & 2454307.7225 & -5.59 & 2454341.5520 & 33.12 & & \\
\hline 2454332.7340 & 30.87 & 2454308.6425 & -4.00 & 2454625.8782 & -4.40 & & \\
\hline 2454333.6771 & 9.67 & 2454309.6342 & 0.67 & 2454626.8872 & -3.10 & & \\
\hline 2454335.6354 & 7.36 & 2454310.7901 & 5.43 & 2454627.6480 & 2.69 & & \\
\hline 2454339.7009 & -0.89 & 2454311.6308 & 11.40 & 2454630.8323 & 35.04 & & \\
\hline 2454341.6982 & 13.71 & 2454312.8179 & 12.64 & 2454631.8732 & 12.36 & & \\
\hline 2454625.9016 & 6.83 & 2454332.7026 & 14.41 & 2454632.8667 & -5.53 & & \\
\hline 2454626.8971 & 16.15 & 2454335.5413 & -5.49 & 2454633.6727 & -0.62 & & \\
\hline 2454627.9075 & 24.67 & 2454336.6798 & -0.42 & 2454634.8377 & 8.53 & & \\
\hline 2454631.7591 & 11.39 & 2454341.6457 & -5.96 & 2454953.7113 & -6.04 & & \\
\hline 2454632.8787 & 20.74 & 2454626.7744 & -1.12 & & & & \\
\hline 2454633.8050 & 29.90 & 2454627.7452 & -7.57 & & & & \\
\hline & & 2454630.8954 & 4.48 & & & & \\
\hline & & 2454633.6873 & -2.61 & & & & \\
\hline
\end{tabular}


Table 4. Published $[\mathrm{Fe} / \mathrm{H}]$ values for the Galactic Cepheids.

\begin{tabular}{|c|c|c|c|}
\hline Name & {$[\mathrm{Fe} / \mathrm{H}]$} & Name & {$[\mathrm{Fe} / \mathrm{H}]$} \\
\hline AK Cep & $+0.05(1)$ & S Vul & $+0.12(1)$ \\
\hline AN Aur & -0.10 (1) & SV Vul & $+0.05(2)$ \\
\hline AQ Pup & $+0.04(1)$ & SW Cas & $+0.13(2)$ \\
\hline AV Sgr & $+0.34(2)$ & SW Vel & $+0.00(1)$ \\
\hline AW Per & $+0.04(1)$ & SX Vel & +0.06 \\
\hline BB Sgr & $+0.08(2)$ & SY Cas & $+0.04(2)$ \\
\hline BE Mon & 0.08 (1) & SZ Aql & $+0.17(2)$ \\
\hline beta Dor & $-0.01(3)$ & SZ Cyg & $+0.09(2)$ \\
\hline BF Oph & $+0.14(1)$ & SZ Tau & $+0.07(2)$ \\
\hline BG Lac & $+0.07(1)$ & T Mon & $+0.23(1)$ \\
\hline BM Per & $+0.23(1)$ & TT Aql & $+0.22(1)$ \\
\hline BN Pup & $+0.11(1)$ & T Vel & +0.04 (1) \\
\hline BN Pup & $+0.11(1)$ & $\mathrm{T} \mathrm{Vul}$ & $+0.01(2)$ \\
\hline BZ Cyg & $+0.19(2)$ & TW Nor & $+0.33(1)$ \\
\hline CD Cyg & $+0.15(1)$ & TY Sct & $+0.37(1)$ \\
\hline CF Cas & $+0.02(1)$ & TZ Mon & $+0.01(1)$ \\
\hline CK Sct & $+0.21(1)$ & U Aql & $+0.17(1)$ \\
\hline СР Cep & $-0.01(2)$ & U Car & +0.04 \\
\hline CR Cep & $-0.06(2)$ & U Nor & +0.19 \\
\hline CS Vel & $+0.12(1)$ & U Sgr & $+0.08(2)$ \\
\hline CV Mon & $+0.01(1)$ & UU Mus & +0.19 \\
\hline DD Cas & $+0.10(2)$ & U Vul & +0.19 (1) \\
\hline delta Cep & $+0.12(1)$ & UZ Sct & $+0.33(2)$ \\
\hline DL Cas & -0.01 (2) & V1162 Aql & $+0.01(2)$ \\
\hline DT Cyg & $+0.10(2)$ & V340 Ara & $+0.31(2)$ \\
\hline eta Aql & $+0.08(2)$ & V340 Nor & $+0.16(1)$ \\
\hline FF Aql & $+0.04(2)$ & V350 Sgr & $+0.18(2)$ \\
\hline FM Aql & $+0.24(1)$ & V386 Cyg & $+0.11(2)$ \\
\hline FM Cas & -0.09 (2) & V402 Cyg & $+0.02(2)$ \\
\hline FN Aql & $-0.06(1)$ & V459 Cyg & +0.09 (1) \\
\hline GH Lup & $+0.13(1)$ & V495 Cyg & $+0.24(1)$ \\
\hline GY Sge & $+0.29(1)$ & V496 Aql & $+0.05(2)$ \\
\hline KN Cen & $+0.41(1)$ & V538 Cyg & +0.05 \\
\hline KQ Sco & $+0.16(2)$ & V600 Aql & $+0.03(2)$ \\
\hline 1 Car & $+0.13(1)$ & V916 Aql & +0.39 (1) \\
\hline LS Pup & $-0.16(3)$ & V Car & +0.04 \\
\hline MW Cyg & $+0.09(2)$ & V Cen & $+0.03(1)$ \\
\hline QZ Nor & $+0.06(4)$ & VW Cen & $-0.02(3)$ \\
\hline RR Lac & $+0.04(1)$ & VX Cyg & $+0.09(2)$ \\
\hline RS Cas & +0.18 & VX Per & $+0.06(1)$ \\
\hline RS Ori & $-0.10(2)$ & VY Car & $+0.02(1)$ \\
\hline RS Pup & $+0.22(1)$ & VY Cyg & $+0.00(2)$ \\
\hline RT Aur & $+0.13(1)$ & VY Sgr & $+0.26(2)$ \\
\hline RU Sct & $+0.11(1)$ & VZ Cyg & $+0.05(2)$ \\
\hline RW Cam & $+0.11(1)$ & VZ Pup & $-0.11(1)$ \\
\hline RW Cas & $+0.22(2)$ & W Gem & $+0.02(1)$ \\
\hline RX Aur & $+0.10(1)$ & W Sgr & $+0.02(2)$ \\
\hline RX Cam & $+0.11(1)$ & WZ Car & $+0.05(1)$ \\
\hline RY Cas & $+0.26(2)$ & WZ Sgr & $+0.19(2)$ \\
\hline RY CMa & $+0.02(1)$ & X Cyg & $+0.10(2)$ \\
\hline RY Sco & $+0.09(2)$ & X Lac & $+0.08(1)$ \\
\hline RY Vel & +0.09 & X Pup & $+0.08(1)$ \\
\hline RZ CMa & -0.03 (1) & X Sgr & $-0.29(5)$ \\
\hline RZ Gem & -0.17 (1) & X Vul & $+0.07(2)$ \\
\hline RZ Vel & $+0.04(1)$ & XX Cen & +0.18 \\
\hline S Mus & +0.07 (1) & XX Sgr & $+0.10(2)$ \\
\hline S Nor & $+0.13(1)$ & Y Lac & $+0.03(1)$ \\
\hline S Sge & $+0.08(2)$ & Y Oph & $+0.06(2)$ \\
\hline SS Sct & +0.14 & Y Sct & $+0.23(1)$ \\
\hline ST Tau & $+0.00(1)$ & Y Sgr & $+0.05(2)$ \\
\hline SU Cas & $+0.06(2)$ & YZ Aur & $-0.30(1)$ \\
\hline SU Cyg & $-0.03(2)$ & YZ Sgr & $+0.06(2)$ \\
\hline SV Mon & $-0.03(2)$ & zeta Gem & +0.10 \\
\hline SV Per & $+0.06(1)$ & Z Lac & $+0.10(1)$ \\
\hline
\end{tabular}

References. 1 = Luck \& Lambert (2011); 2 = Luck et al. (2011); $3=$ Romaniello et al. (2008); 4 = Fry \& Carney (1997); 5 = Andrievsky et al. (2003).

A70, page 20 of 25
Table 5. Published $[\mathrm{Fe} / \mathrm{H}]$ values for the MC Cepheids.

\begin{tabular}{lccc}
\hline \hline Name & {$[\mathrm{Fe} / \mathrm{H}]$} & Name & {$[\mathrm{Fe} / \mathrm{H}]$} \\
\hline HV 837 & $-0.83 \pm 0.10(1)$ & HV 877 & $-0.44 \pm 0.10(1)$ \\
HV 879 & $-0.14 \pm 0.10(1)$ & HV 1023 & $-0.28 \pm 0.10(1)$ \\
HV 2369 & $-0.62 \pm 0.10(1)$ & HV 2405 & $-0.27 \pm 0.10(1)$ \\
HV 2827 & $-0.38 \pm 0.10(1)$ & HV 6093 & $-0.60 \pm 0.10(1)$ \\
HV 12452 & $-0.35 \pm 0.10(1)$ & & \\
HV 12197 & $-0.39 \pm 0.05(2)$ & HV 12199 & $-0.38 \pm 0.06(2)$ \\
HV 900 & $-0.38 \pm 0.10(3)$ & HV 909 & $-0.28 \pm 0.10(3)$ \\
HV 2257 & $-0.34 \pm 0.10(3)$ & HV 2338 & $-0.44 \pm 0.10(3)$ \\
\hline
\end{tabular}

References. 1 = Romaniello et al. (2008); 2 = Molinaro et al. (2012); 3 = Luck \& Lambert (1998). 
M. A. T. Groenewegen: Baade-Wesselink distances and the effect of metallicity in Cepheids

Table 6. Stars with parallaxes and/or interferometrically determined angular diameters.

\begin{tabular}{|c|c|c|c|c|c|c|c|c|c|}
\hline Name & $\begin{array}{l}\text { Period } \\
\text { (days) }\end{array}$ & $E(B-V)$ & $\begin{array}{c}\mathrm{LK} \\
(\mathrm{mag})\end{array}$ & Ref. & $\begin{array}{c}d \\
(\mathrm{pc})\end{array}$ & Ref. & $p$ & $\begin{array}{c}R \\
\left(R_{\odot}\right)\end{array}$ & $p$ \\
\hline T Vul & 4.435421 & 0.064 & -0.09 & 1 & $506 \pm 57$ & 4 & $1.781 \pm 0.421 \pm 0.201$ & $33.85 \pm 0.38 \pm 3.81$ & $1.32 \pm 0.16$ \\
\hline FF Aql & 4.470896 & 0.196 & -0.03 & 1 & $384 \pm 24$ & 4 & $1.534 \pm 0.301 \pm 0.096$ & $36.17 \pm 0.15 \pm 2.26$ & $0.77 \pm 0.15$ \\
\hline delta Cep & 5.366250 & 0.075 & -0.01 & 1,2 & $272.4 \pm 7.3$ & 5,6 & $1.241 \pm 0.030 \pm 0.033$ & $42.43 \pm 0.038 \pm 1.13$ & $1.44 \pm 0.12$ \\
\hline X Sgr & 7.012745 & 0.237 & -0.02 & 1 & $318 \pm 14$ & 7 & $1.025 \pm 0.955 \pm 0.045$ & $49.75 \pm 0.98 \pm 2.19$ & $1.300 \pm 0.087$ \\
\hline W Sgr & 7.594925 & 0.108 & -0.06 & 1 & $447 \pm 38$ & 7 & $1.676 \pm 0.871 \pm 0.143$ & $62.1 \pm 1.6 \pm 5.3$ & $2.83 \pm 0.46$ \\
\hline beta Dor & 9.842554 & 0.052 & -0.02 & 1 & $310 \pm 13$ & 7,8 & $1.439 \pm 0.165 \pm 0.060$ & $61.33 \pm 0.34 \pm 2.57$ & $1.188 \pm 0.062$ \\
\hline zeta Gem & 10.149922 & 0.014 & -0.02 & 1,3 & $361 \pm 11$ & 6,7 & $1.349 \pm 0.106 \pm 0.041$ & $65.39 \pm 0.20 \pm 2.00$ & $1.265 \pm 0.061$ \\
\hline 1 Car & 35.557209 & 0.147 & -0.05 & 1 & $504 \pm 41$ & 9,10 & $1.226 \pm 0.030 \pm 0.100$ & $159.06 \pm 0.27 \pm 12.9$ & $1.30 \pm 0.11$ \\
\hline eta Aql & 7.176814 & 0.130 & - & - & $268 \pm 1.4 \pm 13.9$ & $6,7,8$ & $1.165 \pm 0.148 \pm 0.060$ & $51.3 \pm 2.7$ & - \\
\hline Y Oph & 17.126144 & 0.645 & - & - & $679 \pm 5.1 \pm 36$ & 7 & $1.679 \pm 0.323 \pm 0.087$ & $93.9 \pm 4.9$ & - \\
\hline RT Aur & 5.4820695 & 0.059 & -0.06 & 1 & $445 \pm 38$ & - & & & $1.28 \pm 0.14$ \\
\hline Y Sgr & 5.7644143 & 0.191 & -0.15 & 1 & $503 \pm 75$ & - & & & $1.60 \pm 0.37$ \\
\hline
\end{tabular}

References. (1) van Leeuwen et al. (2007); (2) Majaess et al. (2012a); (3) Majaess et al. (2012b); (4) Gallenne et al. (2012); (5) Merand et al. (2005); (6) Nordgren et al. (2002); (7) Kervella et al. (2004); (8) Jacob (2008); (9) Davis et al. (2009); (10) Kervella et al. (2004b). 
Table 10. Distances, radii, and absolute magnitudes from the BW analysis.

\begin{tabular}{|c|c|c|c|c|c|c|c|}
\hline Name & $E(B-V)$ & Period $(\mathrm{d})$ & $p$ & $d(\mathrm{pc})$ & $R\left(R_{\odot}\right)$ & $M_{\mathrm{V}}$ & $M_{K}$ \\
\hline \multicolumn{8}{|c|}{ Galactic Cepheids } \\
\hline AK Cep & $0.635 \pm 0.049$ & 7.233310 & 1.294 & $\pm 378.2 \pm 628.0$ & $59.5 \pm$ & $-3.90 \pm 0.26 \pm 0.47$ & $-5.52 \pm 0.20 \pm 0.43$ \\
\hline AN Aur & $0.600 \pm 0.057$ & 10.289226 & 1.257 & $5301.6 \pm 544.2 \pm 463.4$ & $93.6 \pm$ & $-5.13 \pm 0.29 \pm 0.31$ & $-6.56 \pm 0.21 \pm 0.20$ \\
\hline AQ Pup & $0.518 \pm$ & 30.095462 & & $2991.1 \pm 80.8 \pm 141.3$ & $137.2 \pm$ & $-5.31 \pm 0.07 \pm$ & $-7.27 \pm 0.06$ \\
\hline$\checkmark$ Sgr & $7 \pm 0.078$ & 15.411587 & 215 & $452.7 \pm 104.0 \pm 198.3$ & $106.2 \pm 4.5 \pm 8.4$ & $-4.77 \pm 0.28 \pm 0.36$ & $-6.66 \pm 0.10=$ \\
\hline AW Per & $9 \pm 0.011$ & 6.463585 & 1.305 & $971.5 \pm 79.4 \pm 93.5$ & $53.9 \pm 4.4 \pm 5.3$ & $-4.06 \pm 0.18 \pm 0.23$ & $-5.41 \pm 0.17$ \\
\hline BB Sgr & $1 \pm 0.009$ & 6.637113 & 1.303 & $814.3 \pm 25.8 \pm 29.0$ & & $-3.53 \pm 0.07 \pm 0.09$ & $-5.15 \pm 0.07 \pm 0.08$ \\
\hline BE Mon & $0.565 \pm 0.038$ & 2.705541 & 1.396 & $1884.0 \pm 283.2 \pm 233.4$ & & $-2.66 \pm 0.33 \pm 0.32$ & $-3.86 \pm 0.30 \pm 0.28$ \\
\hline beta Dor & $0.052 \pm 0.009$ & 9.842554 & 1.262 & & $63.9 \pm 1.2 \pm 1.7$ & $-4.00 \pm 0.05 \pm 0.07$ & $-5.65 \pm 0.04 \pm 0.06$ \\
\hline BF Oph & $0.235 \pm 0.010$ & 4.067677 & 1.354 & $760.2 \pm 32.2 \pm 34.9$ & & $-2.82 \pm 0.10 \pm 0.10$ & $-4.31 \pm 0.09 \pm 0.10$ \\
\hline BG Lac & $0.300 \pm 0.016$ & 5.331921 & 1.326 & $4 \pm 74.4 \pm 78.7$ & $41.9 \pm$ & & $-4.80 \pm 0.09 \pm 0.10$ \\
\hline BM Per & $0.871 \pm 0.048$ & 22.958209 & 1.173 & $\pm 77.1 \pm 740.1$ & $91.9 \pm 3.2 \pm 30.9$ & $-4.16 \pm 0.18 \pm 0.56$ & $-6.33 \pm 0.08 \pm$ \\
\hline BN Pup & $0.416 \pm 0.018$ & 13.672436 & 1.227 & $70.8 \pm 72.8 \pm 95.2$ & $76.3 \pm 1.6 \pm 1.9$ & $-4.18 \pm 0.07 \pm 0.09$ & $-5.99 \pm 0.04 \pm$ \\
\hline BZ Cyg & $0.882 \pm 0.054$ & 10.141721 & 1.259 & $2167.7 \pm 246.1 \pm 251.0$ & $79.4 \pm 9.0 \pm 9.3$ & $-4.36 \pm 0.30 \pm 0.34$ & $-6.10 \pm$ \\
\hline CD Cyg & $0.493 \pm 0.015$ & 17.074070 & 1.204 & $2267.0 \pm 51.1 \pm 83.1$ & $85.9 \pm 2.0 \pm 3.1$ & $-4.40 \pm 0.07 \pm 0.10$ & $-6.26 \pm 0.05 \pm 0.08$ \\
\hline CF Cas & & & & & & & \\
\hline CK Sct & 77 & & & .8 & $60.0 \pm$ & 43 & \\
\hline CP Cep & 050 & 17.8 & .200 & \pm 10 & $2 \pm$ & -4 & \\
\hline R Cep & 016 & & 1.309 & 12 & 8 & 25 & \\
\hline Ser & 887 & 46 & 1.326 & 9.6 & $70.4 \pm 1$ & 54 & - \\
\hline Vel & 29 & & 1.315 & 32.7 & .4 & 17 & \\
\hline Mon & & & & & $40.7=$ & 13 & -4.71 \\
\hline & 016 & 9.8 & 1.2 & 8.3 & & & \\
\hline del Cep & $0.075 \pm 0.009$ & 5.36 & 1.325 & $5+171$ & & & -4 \\
\hline DL Cas & $0.488 \pm 0.010$ & 8.000458 & 1.283 & $\pm 168.9 \pm 182.6$ & $60.7 \pm$ & & 0.23 \\
\hline DT Cyg & $0.042 \pm 0.011$ & $2.4 \mathrm{C}$ & 1.405 & 5.4 & $56.5 \pm$ & & -5 \\
\hline eta Aql & 0.13 & $7.1^{\prime}$ & 1.295 & & & & \\
\hline FF Aql & 0.19 & & & & & & \\
\hline FM Aql & & & & & & & \\
\hline & & & & & & & \\
\hline & & & & & & & \\
\hline Lup & & & & & & & \\
\hline & & & & 1.4 & 157 & & \\
\hline Cen & & & 2 & & & & \\
\hline KQ Sco & 20 & 78 & 0 & .5 & & -4 & -7 \\
\hline $1 \mathrm{Car}$ & & & & & 3.5 & -4 & .06 \\
\hline S Pup & $0.461 \pm 0.015$ & 14.1 & 1.224 & 0.8 & & & -6 \\
\hline MW Cyg & $0.635 \pm 0.017$ & 27 & 1.314 & & & -2 & -4 \\
\hline QZ Nor & $0.253 \pm 0.016$ & 3.78 & 1.3 & $.8 \pm 91.2$ & $32.0 \pm$ & -2 & $-4.20 \pm 0$ \\
\hline RR Lac & $0.319 \pm$ & 6.41 & 1.3 & 126.5 & 41. & -3 . & $-4.79 \pm($ \\
\hline RS Cas & $0.784=$ & 6.29 & & & & -3 . & -4 \\
\hline RS Ori & $0.352 \pm 0.012$ & 7.566893 & 1.289 & 7.6 & & -3 & 0.17 \\
\hline RS Pup & & 711 & & & & & 0.10 \\
\hline RT Aur & & & & & & & \\
\hline & & & & & & & \\
\hline Cam & & & & & & & \\
\hline Cas & & & & & & & \\
\hline Aur & & & & & & & \\
\hline Cam & 11 & & & 5.8 & .1 & $-3.62 \pm 0$ & -5 . \\
\hline RY Cas & 0613. & 39 & 40 & 2.2 & $60.8 \pm$ & $-3.64 \pm 0$ & -5 . \\
\hline & $39 \pm 0.010$ & & & $7 \pm 99.5$ & .1 & \pm 0.19 & -4 \\
\hline RY Sco & $0.718 \pm 0.018$ & 20.321239 & 1.186 & $1087.6 \pm 24.4 \pm 49.9$ & $88.6 \pm 2.0 \pm 4.0$ & $-4.50 \pm 0.08 \pm 0.12$ & $-6.31 \pm 0.05$ \\
\hline RY Vel & $0.547 \pm 0.010$ & 28.124039 & 1.152 & $2002.2 \pm 26.0 \pm 96.1$ & $110.8 \pm 1.4 \pm 5.2$ & $-4.90 \pm 0.04 \pm 0.11$ & $-6.78 \pm 0.03=$ \\
\hline RZ CMa & $0.443 \pm 0.016$ & 4.254980 & 1.349 & $1633.3 \pm 121.6 \pm 133.3$ & $31.3 \pm 2.3 \pm 2.6$ & $-2.81 \pm 0.16 \pm 0.20$ & $-4.21 \pm 0.16 \pm 0.19$ \\
\hline RZ Gem & $0.563 \pm 0.025$ & 5.529040 & 1.322 & $1990.3 \pm 182.5 \pm 193.9$ & $39.2 \pm 3.6 \pm 3.8$ & $-3.28 \pm 0.21 \pm 0.23$ & $-4.70 \pm 0.19 \pm 0.22$ \\
\hline RZ Vel & $0.299 \pm 0.009$ & 20.399697 & 1.186 & $1410.6 \pm 17.3 \pm 38.2$ & $99.8 \pm 1.2 \pm 2.6$ & $-4.58 \pm 0.04 \pm 0.07$ & $-6.54 \pm 0.03 \pm 0.06$ \\
\hline S Mus & $0.212 \pm 0.017$ & 9.659971 & 1.264 & $820.6 \pm 33.3 \pm 31.1$ & $61.7 \pm 2.5 \pm 2.4$ & $-4.12 \pm 0.10 \pm 0.10$ & $-5.64 \pm 0.09 \pm 0.09$ \\
\hline S Nor & $0.179 \pm 0.009$ & 9.754255 & 1.263 & $814.1 \pm 23.0 \pm 28.4$ & $59.5 \pm 1.7 \pm 2.1$ & $-3.70 \pm 0.07 \pm 0.08$ & $-5.46 \pm 0.06 \pm 0.07$ \\
\hline S Sge & $0.100 \pm 0.010$ & 8.382073 & 1.278 & $680.2 \pm 18.1 \pm 20.8$ & $56.8 \pm 1.5 \pm 1.8$ & $-3.85 \pm 0.07 \pm 0.08$ & $-5.44 \pm 0.06 \pm 0.07$ \\
\hline SS Sct & & & 1.364 & $394.4 \pm 130.8 \pm 111.2$ & $45.6 \pm 4.3 \pm 3.6$ & $-3.58 \pm 0.20 \pm 0.20$ & $-5.00 \pm 0.19 \pm 0.20$ \\
\hline ST Tau & $0.368 \pm 0.030$ & 4.034249 & 1.355 & $1174.3 \pm 118.7 \pm 115.0$ & & $-3.32 \pm 0.23 \pm 0.25$ & $-4.69 \pm 0.21$ \\
\hline U Cas & & & 1.430 & $425.7 \pm 26.1 \pm 27.6$ & & $-3.03 \pm 0.13 \pm 0.15$ & $-4.12 \pm 0.13$ \\
\hline & & & & & $37.4 \pm 2.3 \pm 2.0$ & $-3.34 \pm 0.14=$ & $-4.63 \pm 0.13$ \\
\hline & & & & & & & $-5.84 \pm 0$ \\
\hline & & & & & & & \\
\hline S Vul & $0.727 \pm 0.043$ & 8.711152 & 1.059 & $7 \pm 195.8$ & $272.4 \pm$ & & \\
\hline
\end{tabular}


M. A. T. Groenewegen: Baade-Wesselink distances and the effect of metallicity in Cepheids

Table 10. continued.

\begin{tabular}{|c|c|c|c|c|c|c|c|}
\hline ne & $E(B-V)$ & $\operatorname{eriod}(d)$ & $p$ & $d(\mathrm{pc})$ & $R\left(R_{\odot}\right)$ & $M_{\mathrm{V}}$ & $M_{K}$ \\
\hline SV Vul & $461 \pm 0.021$ & 5.027988 & .103 & $2173.5 \pm 53.1 \pm 72.2$ & $89.2 \pm 4.6 \pm 8.2$ & $-5.95 \pm 0.09 \pm 0.11$ & $-7.93 \pm 0.05 \pm 0$ \\
\hline Cas & $67+0.018$ & 909 & 1.323 & $2126.2 \pm 202.8 \pm 219.4$ & $45.1 \pm 4.3 \pm 4.8$ & $-3.45 \pm 0.21 \pm 0.25$ & $-4.96 \pm 0.20$ \\
\hline & & & & & & $-4.54+$ & $-6.49 \pm$ \\
\hline & & & & 1408 & & & \\
\hline & & & 354 & 1870 & 3.2 & $-2.85 \pm 0.17 \pm 0.27$ & $-4.18+$ \\
\hline & & & 204 & & & .09 & -6.30 \\
\hline SZ Cyg & & 10 & 1.217 & 2320 & & .12 & $-6.27+$ \\
\hline & & & 1.380 & & & .11 & $-4.69+$ \\
\hline & 011 & 992 & 1.156 & & 3.4 & $-4.67 \pm$ & $-6.79 \pm$ \\
\hline & .011 & 828 & 1.227 & & & $-4.24 \pm 0.08 \pm 0.08$ & $-6.08 \pm 0$. \\
\hline T Vel & 009 & & 1.340 & & & $-2.86 \pm 0$ & $-4.41 \pm 0.02$ \\
\hline & & & 1.345 & & $.7 \pm$ & $-2.99 \pm 0.06 \pm 0.07$ & $-4.37 \pm 0.05 \pm 0.06$ \\
\hline W Nor & 013 & 356 & 1.252 & 2293 & .4 & $-3.92 \pm$ & $-5.84 \pm$ \\
\hline Y Sct & & & 1.250 & 2002 & & $-3.74 \pm$ & $-5.44 \pm$ \\
\hline & & & & 432 & & .23 & \\
\hline & & & & & & & \\
\hline & & & & & & & \\
\hline & & & & & & & \\
\hline & & & & & & 09 & \\
\hline & & & & & & 12 & \\
\hline & & & 283 & & & 12 & - \\
\hline & & & 220 & & & 27 & .11 \\
\hline-2 & & & 25 & & & .46 & -4.84 \\
\hline & & & & & & .23 & \\
\hline & & & & & & & \\
\hline Sor & & & 1.329 & & & & -4 \\
\hline $\mathrm{Cyg}$ & & & & & & & -4 . \\
\hline Cyg & & & & & & 23 & -4 \\
\hline & & & & & & & -4. \\
\hline & & & & & & & \\
\hline & & & & & & & \\
\hline & & & & & & & \\
\hline & & & & & & & -5 . \\
\hline 政 & & & & & & & \\
\hline & & & & & & 13 & -2 \\
\hline & & & & & & 09 & -4 . \\
\hline & & & & & & 13 & -6 . \\
\hline & & & & & & & \\
\hline & & & & & 3.8 & 28 & -6. \\
\hline & & & & & & -3. & -5 . \\
\hline & & & & & & & -4. \\
\hline & & & & & & 32 & -6.5 \\
\hline & & & & & & & -4. \\
\hline & & & & & & & \\
\hline & & & & & & & \\
\hline & & & & & & & \\
\hline & & & & & & & \\
\hline & & & & & & & \\
\hline & & & & & & & \\
\hline & & & & & & 13 & -5 \\
\hline & & & & & & 10 & -6 . \\
\hline & & & & & & & \\
\hline & & & & 8 & 0 & $-3.29 \pm$ & $-4.73 \pm$ \\
\hline & & & & & \pm & $-3.76 \pm$ & $-5.42 \pm$ \\
\hline & & & & & 7 & -3. & $-4.99 \pm$ \\
\hline & & & & 2 & .4 & $-3.66 \pm$ & $-5.00 \pm$ \\
\hline $\mathrm{Y}$ & & & & - & .0 & $-4.70 \pm$ & $-6.30 \pm 0$ \\
\hline & 12 & 10.3 & 1.257 & 1770 & $71.1 \pm$ & $-4.08 \pm 0.14 \pm 0.15$ & $-5.84 \pm 0.13 \pm 0.15$ \\
\hline & & & & & & & $-4.55 \pm$ \\
\hline & & & & & & & \\
\hline YZ Sgr & & & & & & & \\
\hline & & & & & & $-3.93 \pm$ & $-5.65 \pm$ \\
\hline & $0.370 \pm 0.011$ & 10.885697 & 1.251 & $1813.0 \pm 44.0 \pm 70.1$ & $67.0 \pm 1.6 \pm 2.6$ & $-4.06 \pm 0.06 \pm 0.10$ & $-5.74 \pm 0.05 \pm 0.09$ \\
\hline
\end{tabular}


Table 10. continued.

\begin{tabular}{|c|c|c|c|c|c|c|c|}
\hline Name & $E(B-V)$ & Period (d) & $p$ & $d(\mathrm{pc})$ & $R\left(R_{\odot}\right)$ & $M_{\mathrm{V}}$ & $M_{K}$ \\
\hline \multicolumn{8}{|c|}{ LMC Cepheids } \\
\hline HV 1005 & $0.100 \pm 0.005$ & 18.714651 & 1.195 & $44096.6 \pm 1787.4 \pm 1141.7$ & $82.6 \pm 3.4 \pm 2.1$ & $-4.39 \pm 0.09 \pm 0.06$ & $-6.20 \pm 0.09 \pm 0.06$ \\
\hline HV 1006 & $0.100 \pm 0.005$ & 14.216644 & 1.223 & $2626.4 \pm 1557.0 \pm 3293.9$ & $73.9 \pm 2.7 \pm 5.3$ & $-4.04 \pm 0.08 \pm 0.17$ & $-5.90 \pm 0.08 \pm 0.16$ \\
\hline HV 1023 & $0.070 \pm 0.005$ & 26.554194 & 1.158 & $48109.6 \pm 1770.2 \pm 2438.1$ & $120.3 \pm 4.5 \pm 5.5$ & $-4.81 \pm 0.08 \pm 0.12$ & $-6.92 \pm 0.08 \pm 0.12$ \\
\hline HV 12197 & $0.060 \pm 0.005$ & & 1.381 & $519.9 \pm 2388.9 \pm 1541.2$ & $22.6 \pm 1.4 \pm 0.9$ & & $-3.48=$ \\
\hline HV 12198 & $0.060 \pm$ & 3.522766 & 1.369 & $786.8 \pm 2073.1$ & $30.2=$ & -2.6 & -4.10 \\
\hline HV 12199 & $0.060 \pm$ & 2.639167 & 1.399 & $17.8 \pm 3516.8 \pm 3040.6$ & 1.6 & $-2.46 \pm 0.14 \pm 0.14$ & $-3.87 \pm 0.14$ \\
\hline HV 12202 & $0.060 \pm$ & 3.101216 & 1.382 & $765.2 \pm 2292.9 \pm 2234.2$ & $21.7 \pm$ & $-1.89 \pm 0.14 \pm 0.14$ & $-3.38 \pm 0$ \\
\hline HV 12203 & $0.060 \pm 0.004$ & 2.954123 & 1.387 & $902.6 \pm 2957.3 \pm 2481.8$ & $24.8 \pm 1.6 \pm 1.4$ & $-2.32 \pm 0.14 \pm 0.14$ & $-3.70 \pm 0.14$ \\
\hline HV 12204 & $0.060 \pm 0.005$ & 3.43 & 1.371 & $9 \pm 2154.3 \pm 3331.3$ & & $-2.75 \pm 0.10 \pm 0.17$ & $-4.04 \pm 0.10 \pm 0.17$ \\
\hline HV 12452 & $0.058 \pm 0.005$ & 8.738897 & 1.274 & $39620.7 \pm 1448.4 \pm 3968.2$ & & $-3.39 \pm 0.08 \pm 0.22$ & $-5.06 \pm 0.08 \pm 0.22$ \\
\hline HV 12505 & $0.100 \pm 0.005$ & 14.393280 & 1.222 & $46661.2 \pm 1577.9 \pm 4095.0$ & & $-3.79 \pm 0.07 \pm 0.19$ & $-5.90 \pm 0.07 \pm 0.19$ \\
\hline HV 12717 & $0.058 \pm 0.005$ & & 1.273 & & & $-3.18 \pm 0.19 \pm 0.23$ & $-4.78 \pm 0.18 \pm 0.23$ \\
\hline HV 12815 & $0.070 \pm 0.005$ & 26.115120 & 1.160 & $39034.2 \pm 2303.9 \pm 3812.0$ & $105.2 \pm 6.2 \pm 11.0$ & $-4.65 \pm 0.13 \pm 0.21$ & $-6.66 \pm 0$ \\
\hline HV 12816 & $0.070 \pm 0.005$ & 9.108991 & 1.270 & $26.6 \pm 3305.1 \pm 3137.1$ & $53.5 \pm 3.8 \pm 3.6$ & $5 \pm 0.16$ & $-5.38 \pm$ \\
\hline HV 2257 & $0.060 \pm 0.005$ & 39.388561 & 1.117 & $3.9 \pm 1085.6 \pm 2119.3$ & $156.6 \pm 3.7 \pm 6.5$ & $-5.33 \pm 0.05 \pm 0.10$ & $-7.47 \pm$ \\
\hline HV 2282 & $0.100 \pm 0.005$ & 14.677123 & 1.220 & $44678.3 \pm 1082.1 \pm 1790.5$ & & $-4.20 \pm 0.05 \pm 0.09$ & \\
\hline & $0.040 \pm 0.005$ & 42.1 & 1.110 & & & $-5.48 \pm 0.04 \pm 0.10$ & \\
\hline HV 2369 & \pm 0.005 & 48.3 & 1.096 & & $151.2 \pm$ & .13 & \\
\hline & 005 & & 1.2 & 5.9 & & 25 & -5 \\
\hline 27 & 0.07 & 12.9 & 233 & $1 \pm 2326.8 \pm 2$ & 3.4 & .11 & $-5.89 \pm 0$ \\
\hline $\mathrm{HV}$ & 005 & 13.8 & 26 & 441. & $79.8 \pm$ & $-4.25 \pm 0$ & -6.07 \\
\hline $\mathrm{HV}$ & 05 & 20 & 1.210 & \pm 2762.6 & $86.7 \pm$ & $-4.64=$ & $-6.32 \pm$ \\
\hline HV 2827 & $0.080 \pm$ & 78.824495 & 1.045 & $\pm 1652.4 \pm 1721.4$ & $222.2 \pm 9.0 \pm 9.6$ & $-6.00 \pm 0$ & $-8.21 \pm 0$ \\
\hline HV 5655 & $0.100 \pm$ & 14.212586 & 1.223 & $45859.2 \pm 2057.8 \pm 3650.0$ & $7 \pm 3.3 \pm 5.3$ & $5 \pm 0.10 \pm 0.18$ & $-5.88 \pm 0.1$ \\
\hline HV 6093 & $0.058 \pm 0.005$ & 4.784880 & 1.337 & $47300.7 \pm 3548.0 \pm 4795.1$ & $37.2 \pm 2.8 \pm 3.7$ & $-3.23 \pm 0.16 \pm 0.22$ & $-4.59 \pm 0.16 \pm 0.22$ \\
\hline HV 873 & $0.130 \pm 0.005$ & 34.436191 & 1.131 & $50117.9 \pm 1128.8 \pm 2200.7$ & $148.5 \pm 3.4 \pm 6.7$ & $-5.84 \pm 0.05 \pm 0.10$ & $-7.53 \pm 0.05 \pm 0.10$ \\
\hline HV 876 & $0.100 \pm 0.005$ & 22.715624 & 1.174 & $44756.6 \pm 1588.5 \pm 1883.9$ & $97.4 \pm 3.5 \pm 3.8$ & $-4.86 \pm 0.08 \pm 0.09$ & $-6.60 \pm 0.08 \pm 0.09$ \\
\hline HV 877 & $0.100 \pm 0.005$ & 45.158119 & 1.103 & $49137.9 \pm 2137.0 \pm 5164.0$ & $171.7 \pm 7.5 \pm 18.4$ & $-5.41 \pm 0.09 \pm 0.27$ & $-7.64 \pm 0.09 \pm 0.27$ \\
\hline HV 878 & $0.058 \pm 0.005$ & 23.306146 & 1.172 & $49845.2 \pm 1349.1 \pm 1707.2$ & $111.2 \pm 3.0 \pm 3.9$ & $-5.06 \pm 0.06 \pm 0.08$ & $-6.85 \pm 0.06 \pm 0.07$ \\
\hline HV 879 & $0.060 \pm 0.005$ & 36.831567 & 1.124 & $43030.6 \pm 2890.7 \pm 2305.0$ & $131.8 \pm 8.9 \pm 7.3$ & $-4.94 \pm 0.14 \pm 0.12$ & $-7.13 \pm 0.14 \pm 0.12$ \\
\hline HV 881 & $0.030 \pm 0.005$ & 35.743231 & 1.127 & $40336.0 \pm 0949.4 \pm 1065.7$ & $121.2 \pm 2.9 \pm 3.2$ & $-4.96 \pm 0.05 \pm 0.06$ & $-6.97 \pm 0.05 \pm 0.06$ \\
\hline HV 899 & $0.110 \pm 0.005$ & 31.050706 & 1.142 & $47670.4 \pm 1231.5 \pm 1397.6$ & $128.0 \pm 3.3 \pm 3.8$ & $-5.34 \pm 0.06 \pm 0.07$ & $-7.16 \pm 0.06 \pm 0.07$ \\
\hline & $0.058 \pm 0.005$ & 47.481696 & 1.098 & $45391.7 \pm 0988.8 \pm 1358.3$ & $165.0 \pm 3.6 \pm 4.7$ & $-5.65 \pm 0.05 \pm 0.07$ & $-7.65 \pm 0.05 \pm 0.07$ \\
\hline & $0.058 \pm 0.005$ & 37.558988 & 1.122 & $40579.0 \pm 1215.3 \pm 1$ & 5.0 & $-5.40 \pm 0$ & $-7.16 \pm 0.06 \pm 0.09$ \\
\hline HV 914 & 0.07 & 6.878394 & 1.299 & $356.1 \pm 5016.2 \pm 2$ & 2.8 & $-3.81 \pm 0.20 \pm 0.12$ & $-5.32 \pm 0.20 \pm 0.12$ \\
\hline 1 & $0100+0005$ & 22.542693 & 1175 & $\begin{array}{r}52497.2 \pm 1546.8 \pm 2528.2 \\
\text { SMC Cepheids }\end{array}$ & $113.2 \pm 3.4 \pm 5.1$ & $-4.76 \pm 0.07 \pm 0.11$ & $-6.82 \pm 0.06 \pm 0.10$ \\
\hline HV 1345 & 0.03 & 13. & 1.229 & $41067.2 \pm 1705.7 \pm 1484.6$ & 5 & 0.09 & -5.22 \\
\hline HV 1335 & & & & & & $-3.68=$ & $-5.23 \pm 0.09 \pm 0.11$ \\
\hline HV 1328 & $0.004 \pm 0.003$ & 15.835971 & 1.212 & $55005.4 \pm 3950.6 \pm 4054.3$ & $77.2 \pm 5.5=$ & $-4.57 \pm 0.15 \pm 0.16$ & $-6.11 \pm 0.15 \pm 0.16$ \\
\hline HV 1333 & $0.070 \pm 0.005$ & 16.295258 & 1.209 & $65988.1 \pm 2898.9 \pm 4126.9$ & $85.9 \pm 3.8 \pm 5.6$ & $-4.59 \pm 0.09 \pm 0.14$ & $-6.29 \pm 0.09 \pm 0.14$ \\
\hline HV \& & $0.030 \pm 0.005$ & 16.742306 & 1.206 & $67441.1 \pm 2403.2 \pm 3533.0$ & $97.2 \pm 3.5 \pm 5.4$ & $-4.66 \pm 0.08 \pm 0.12$ & $-6.52 \pm 0.08 \pm 0.12$ \\
\hline HV 837 & $0.042 \pm 0.005$ & 42.705045 & 1.109 & $56363.4 \pm 3097.9 \pm 2949.2$ & $162.1 \pm 8.9 \pm 8.5$ & $-5.61 \pm 0.12 \pm 0.12$ & $-7.59 \pm 0.12 \pm 0.12$ \\
\hline
\end{tabular}




\section{Appendix A: Cluster distances}

It is not our intention to give a review on the distances to Galactic clusters containing Cepheids. Recent compilations of distances can be found in Turner (2010) and Tammann et al. (2003). The latter is based on Feast (1999), which is an update of Feast \& Walker (1987), with detailed remarks in Walker (1987b).

However, when comparing cluster distances quoted in the literature to the BW distances obtained here and checking the literature in more detail, it was obvious that not all distances were given on the same distance scale. In addition, some results obtained since Turner (2010) can be included in the analysis.

Traditionally, the distances to clusters are based on Zero Age Main Sequence (ZAMS) fitting using $B V$ data and a reference ZAMS, which is very often that of Turner (1976, 1979a). It is tied to a DM to the Pleiades of 5.56.

Recently, Turner, Majaess and co-workers used ZAMS fitting with 2MASS JHK data to derive distances to Cepheids containing clusters. The procedure is outlined in Majaess et al. (2011), and the distances to nine benchmark open clusters thathave HST and revised HIPPARCOS-based distances (van Leeuwen 2009) determined. There is the well-known disagreement for the Pleiades, but the infrared ZAMS fitting distances to the other clusters, and the comparison to the Hyades and Pleiades that have HST-based parallaxes is excellent. The 2MASS-based ZAMS fitting is therefore tied to a DM of 5.65 for the Pleiades, which is thus different from that implied when using the Turner ZAMS in the optical.

In Table A.1, the adopted cluster-based DM are listed for the Cepheids in the sample. Infrared ZAMS fitting is preferred over earlier work in the optical. Where appropriate, the older work is scaled to the adopted Pleiades distance. A few Cepheids in clusters that are in our sample, but where the association is uncertain or the DM in the literature are very discrepant, have not been considered: KQ Sco, GY Sge, T Mon, SV Vul (see Hoyle et al. 2003)

Table A.1. Distances to cluster containing Cepheids.

\begin{tabular}{|c|c|c|c|c|c|}
\hline Name Cepheid & Name Cluster & Adopted DM & Method & Reference & Remarks \\
\hline BB Sgr & Collinder 394 & $9.38 \pm 0.10$ & JHK & Turner (2010) & \\
\hline V Cen & NGC 5662 & $9.28 \pm 0.05$ & JHK & Turner (2010) & \\
\hline RU Sct & Trumpler 35 & $11.11 \pm 0.10$ & JHK & Turner (2010) & \\
\hline SU Cyg & Turner 9 & $9.33 \pm 0.05$ & JHK & Turner (2010) & \\
\hline S Vul & Anon Vul OB & $12.47 \pm 0.29$ & JHK & Turner (2011) & \\
\hline delta Cep & Cep OB6 & $7.21 \pm 0.13$ & JHK & Majaess et al. (2012a) & \\
\hline zeta Gem & ADS 5742 & $7.75 \pm 0.09$ & JHK & Majaess et al. (2012b) & \\
\hline SU Cas & Alessi 95 & $8.04 \pm 0.08$ & JHK & Majaess et al. (2012c) & \\
\hline VY Car & Car OB2 & $11.66 \pm 0.15^{*}$ & BV & Turner (1977) & includes a +0.09 correction in DM \\
\hline RZ Vel & Vel OB1 & $11.32 \pm 0.15^{*}$ & BV & Turner (1979b) & includes a +0.09 correction in DM \\
\hline CS Vel & Ruprecht 79 & $12.55 \pm 0.16$ & BV & Walker (1987c) & includes a +0.08 correction in $\mathrm{DM}$ \\
\hline SZ Tau & NGC 1647 & $8.76 \pm 0.02$ & BV & Turner (1992) & includes a +0.09 correction in DM \\
\hline SW Vel & Vel OB 5 & $12.08 \pm 0.15$ & BV & Turner et al. (1993) & includes a +0.09 correction in DM \\
\hline X Cyg & Ruprecht 175 & $10.52 \pm 0.04$ & BV & Turner (1998) & includes a +0.09 correction in DM \\
\hline USgr & IC 4725 & $9.05 \pm 0.09$ & BV & $a$ & $a$ \\
\hline DL Cas & NGC 129 & $11.10 \pm 0.07$ & BV & $b$ & $b$ \\
\hline S Nor & NGC 6087 & $9.82 \pm 0.18$ & $\mathrm{BV}$ & $c$ & $c$ \\
\hline TW Nor & Lynga 6 & $11.40 \pm 0.12$ & BV & $d$ & $d$ \\
\hline QZ Nor, V340 Nor & NGC 6067 & $11.15 \pm 0.09$ & $\mathrm{BV}$ & $e$ & $e$ \\
\hline CV Mon & vandenBergh 1 & $11.12 \pm 0.15$ & $\mathrm{BV}$ & $f$ & $f$ \\
\hline WZ Sgr & Turner 2 & $11.31 \pm 0.10$ & BV & $g$ & $g$ \\
\hline CF Cas & NGC 7790 & $12.63 \pm 0.11$ & BV & $h$ & $h$ \\
\hline
\end{tabular}

Notes. ${ }^{(a)}$ The average of the distances quoted in An et al. (2007; $8.93 \pm 0.08$ plus a +0.02 correction), Hoyle et al. $(2003 ; 9.08 \pm 0.18$ plus a +0.09 correction), and Pel et al. $\left(1985 ; 8.95 \pm 0.10\right.$ plus a +0.08 correction). ${ }^{(b)}$ The average of the distances quoted in Turner et al. (1992; $11.11 \pm 0.02$ plus a +0.09 correction), An et al. (2007; $11.04 \pm 0.05$ plus a +0.02 correction), and Hoyle et al. $(2003 ; 10.94 \pm 0.14$ plus a +0.09 correction). (c) The average of the distances quoted in Turner (1986; $9.78 \pm 0.03$ plus a +0.09 correction), An et al. (2007; $9.65 \pm 0.06$ plus a +0.02 correction), and Pel et al. (1985; $9.84 \pm 0.10$ plus a +0.08 correction). ${ }^{(d)}$ The average of the distances quoted in An et al. $(2007 ; 11.51 \pm 0.13$ plus a +0.02 correction), Hoyle et al. $\left(2003 ; 11.33 \pm 0.18\right.$ plus a +0.09 correction), and Walker et al. $\left(1985 \mathrm{a} ; 11.15 \pm 0.3\right.$ plus a +0.09 correction). ${ }^{(e)}$ The average of the distances quoted in An et al. $(2007 ; 11.03 \pm 0.08$ plus a +0.02 correction), Hoyle et al. $(2003 ; 11.18 \pm 0.12$ plus a +0.09 correction), and Walker et al. (1985b; $11.05 \pm 0.10$ plus a +0.09 correction). ${ }^{(f)}$ Three distance determinations have been considered: Turner et al. (1998; $11.08 \pm 0.03$ plus a +0.09 correction, adopting $E(B-V)=0.75)$, An et al. $(2007 ; 10.74 \pm 0.21$ plus a +0.02 correction, adopting $E(B-V)=0.57)$, and Hoyle et al. $(2003 ; 11.34 \pm 0.21$ plus a +0.09 correction, adopting $E(B-V)=0.90)$. The adopted distance is the average of the three, but the dispersion is large. This is likely due to the very different reddenings adopted. If a correction is made to a reddening of 0.75 , adopting $\Delta \mathrm{DM} / \Delta$ $\mathrm{E}(\mathrm{B}-\mathrm{V}) \sim 2$ (An et al. 2007), then the average becomes 11.14 with a very small dispersion. ${ }^{(g)}$ The average of the distances quoted in Turner et al. $\left(1993 ; 11.26 \pm 0.10\right.$ plus a +0.09 correction), and Hoyle et al. $\left(2003 ; 11.18 \pm 0.16\right.$ plus a +0.09 correction). ${ }^{(h)}$ The average of the distances quoted in An et al. (2007; $12.46 \pm 0.11$ plus a +0.02 correction), Hoyle et al. (2003; $12.58 \pm 0.14$ plus a +0.09 correction), and Romeo et al. (1989; $12.65 \pm 0.15$ plus a +0.08 correction). ${ }^{(*)}$ No error quoted, conservative error adopted. 